\title{
Stochastic approach to worldwide language classification: the signals and the noise towards long-range exploration
}

\author{
Beaufils Vincent ${ }^{1}$, Tomin Johannes ${ }^{2}$, \\ 1 Independent researcher, Vienna, Austria \\ 2 Independent researcher, Vienna, Austria
}

\begin{abstract}
The exploration of distant language relationships reaching back to the Neolithic age remains very demanding and is often perceived as controversial. Over the last two decades, significant advances in computational linguistics offer alternatives to the methods in use so far to classify languages, but less quantitative research has been devoted to long-range language relationships. Classical methods reach their limits at a level where the signals they infer are more likely to be due to chance than to relatedness. We created a distance-based method without dependency on human relatedness judgments to set up an automated language classification inference system. We used a backward selection method to optimize the key elements of the system towards existing reference classifications: a word list, sound change rules and other parameters. We arbitrated choices in study design and features, which are beneficial for longer-range inference, if necessary at the cost of the short-range performance. The scope of the project is global: the system processes 1962 languages from all families, the limit for inclusion of more languages being the availability of the material needed by our system. After exploring the methodology, we show that it leads to a reliable language classification, matching the reference listings to a high proportion. We then apply a series of statistical models to target middle- and long-range relationships and explore connections outside the well-established classifications. By shedding light on relationships between top-level language families in the context of chance interference, our results deliver a strong support for two major long-range hypotheses: Eurasiatic (connecting Indo-European, Uralic, Turkic, Mongolic and Tungusic) and Austric (connecting Austronesian, Tai-Kadai, Austroasiatic and Hmong-Mien). Further support is given to parts of the Nostratic macrofamily, to a dozen other long-range hypotheses and to internal classifications of established language families.
\end{abstract}

\section{Introduction}

Retracing the origin of our languages and building their family trees gives a fascinating glance at the past, far beyond recorded history. Throughout the 19th and 20th centuries, classical methods in comparative linguistics like the reconstruction of proto-languages have delivered a very high level of consensus about the classification of languages in top-level families (TLF) and their subfamilies, but many aspects of these classifications remain unknown and the number of TLF or primary language families is high. The term "primary language family" does not necessarily imply that no earlier relationship exists between the established ones. Ideally, with further progress in the fields, the number of primary language families will become smaller overtime. When it 
comes to infer connections deeper in time, the classical methods reach their limits: the available data is too eroded for these methods to fully apply. For this reason, in the research of long-range relationships between languages, proto-language reconstruction is not the main activity. The search for relatedness signals is widely extended to related meanings and roots with semantic proximity for various concepts, which seem to share similarities. Authors like Aharon Dolgopolsky, V.M. Illych-Svitych, Josef H. Greenberg, Allan R. Bomhard to name but a few have compiled big lists of such evidence, which lead to hypotheses for macrofamilies linking established top-level families, including reconstructed roots for very old proto-languages. The most discussed hypotheses Eurasiatic and Nostratic - have gathered abundant publications and today, it is less the recognition of their existence which is at stake but more their exact composition. The Moscow School of Comparative Linguistics and its Global Lexicostatistical Database [1] are the leading reference in long-range research. A major point of criticism of long-range hypotheses is that their evidence can be due to chance: the researchers gather clues for hypotheses by extending the possible list of cognates to synonyms and more or less clearly semantically related concepts. By doing so, they increase the exposure to chance.

An alternative can be seen in quantitative approaches. First large-scale quantitative methods around the work of Mauricio Swadesh from the 1950s onwards are known under the terms "lexicostatistics" and "glottochronology". They have been regarded mostly as controversial by mainstream historical linguists and the revival of quantitative methods in the 2000s cannot be seen as their continuity. The possibilities new technologies bring along have offered historical linguists not only methods for automatic word comparisons [2] but also a wider set of mathematical techniques, including Bayesian inference and machine learning, most of the time adapted from biology and genetics 3 . These techniques have proven to be reliable for short- and medium-range classifications, with a first global Automated Classification of the World's Languages (Brown, C. H., E. W. Holman, S. Wichmann, and V. Velupillai) [4] in 2008. Further research in the past 10 years have made considerable steps forward, with Gerhard Jäger's Global-scale phylogenetic linguistic inference from lexical resources $[5]$ as a key example of a successful computer-assisted language classification in 2018. The ERC-funded research project "CALC" (Computer-Assisted Language Comparison) aims at "reconciling classical and computational approaches in historical linguistics" and pushes forward a productive use of computational approaches. Attempts to use mathematical and computer techniques for long-range exploration are increasing, however they do not lead to converging results yet. We have mentioned that long-range research needs to adapt to fading signals in classical approaches. The same applies to long-range research in computational historical linguistics and this aspect is the main focus of our research.

Our key idea is that the use of a quantitative approaches to explore long-range language relationships should address chance as big statistical noise in an environment with very few signals. We are not looking for signals that support specific hypotheses: we create a system which delivers a huge number of results along with the probability that these results and better ones are due to chance. In this context, the system infers possible candidates for long-range hypotheses and we check if such hypotheses already exist in the last step. Clusters of results linking more than just pairs of top-level families are statistically particularly convincing. Designing a system, dedicated to long-range exploration cannot be done without first proving it also works well on shorter-range classifications. For this reason, a big part of the research is devoted to the conception and calibration of the system towards an ideal performance on the classifications which are not contested among scholars. This represents the first step in the project. With a clear focus on the ability to detect longer-range signals and powerful features to challenge results with chance, we have managed to shed light on a 
number of long-range hypotheses from a probabilistic perspective: Nostratic, Austric as the bigger ones, as well as support for smaller macrofamilies in the Americas and Caucasus. Support is also given to the Mande - Niger-Congo and West Bomberai Timor-Alor-Pantar - Trans-New Guinea connections. It is important to point out that our focus is on probabilistic issues. The distinction between relatedness and potential early language contacts is a separate issue, which leaves uncertainity over final judgement about macrofamilies of languages.

\section{Materials and methods}

\section{Word encoding}

To handle comparisons, we encode the lexical items to make them computer-readable. We use the ASJP database 8] as source of lexical items for all languages. The encoding rules have to be adapted to the material available for a wide range of languages. In well-studied language, a high degree of precision of the pronunciation is available. In other languages, only approximations are at our disposal. A central requirement is to use the same rules all over the system. For this reason, we choose a level of precision for the word encoding, which is adapted to the material we have at our disposal from various sources, using the same accuracy degree.

\section{System based on consonants}

We make the choice to keep only consonants - and exclude vowels. This choice is a key point in the design of this research. The higher stability of consonants 6 is a good condition to retrace medium- and long-range relationships. In some cases, vowels resist longer to change than consonants do, but on a global scale, our experience hints at greater value of vowels for short-range comparisons - their value decreasing with the researched time depth. In few cases, most notably in the classification within the Polynesian subfamily of Austronesian, which has been very conservative in its vowel systems 7], the lack of vowel assessment leads to a misclassification within the subfamilies. These biases certainly affect - to various extends - our results in other language groups as well. This weakness is the manageable downside in the search for a simple and universal system. As a second reason to keep vowels out is that their inventory is smaller than the consonants' inventory. The ASJP Database [8] uses 33 consonant symbols versus only 7 vowels symbols, so using vowels brings a bigger exposure to chance than using consonants. The third reason is the lack of their availability in some sources, especially for ancient languages.

\section{Grouping consonants into classes}

There are dozens of consonants in use in world languages - with languages reported to use up to 122 consonants 9. However, for most languages, modern and ancient, only a sound approximation is available in the sources. It is neither possible to codify the available material in 1962 languages according to the very detailed IPA classification nor does it make sense: slight nuances determine the difference between numerous consonants. At the other end of modelling possibilities are Dolgopolsky's ten fundamental sound classes, which deliver few false positive observations in cognate search, but miss potential for finding enough cognates [10]. Matching consonant classes in further quantitative research [11] also tends to use bigger classes. To balance between the extremes, we group the consonants into 27 classes. Consonants share the same class when they differ only slightly from each other, underlying similar change rules towards other classes. Our consonant classification system is summarized in Fig 1. 
Fig 1. The consonant classification used in our research.

\begin{tabular}{|c|c|c|}
\hline Consonant class & ASJP equivalent & IPA code of included sounds \\
\hline -B- & $b$ & $B, \beta, b$ \\
\hline$-\mathrm{C}-$ & c & $\mathrm{ts}, \mathrm{dz}$ \\
\hline$-\mathrm{CH}-$ & $\mathrm{C}, \mathrm{T}$ & $t\left[, t^{j},, c, t\right.$ \\
\hline -D- & d & $d, d^{6}, d, d$ \\
\hline$-\mathrm{F}-$ & $f$ & $f$ \\
\hline -G- & $\mathrm{g}$ & $g, t, G$ \\
\hline$-\mathrm{H}-$ & h & $h, h$ \\
\hline$-\mathrm{A}-$ & $y$ & i \\
\hline$-\mathrm{K}-$ & $k, q$ & $k, q$ \\
\hline$-\mathrm{KH}-$ & $\mathrm{X}, \mathrm{x}$ & $x, r, \hbar, x, x$ \\
\hline$-\mathrm{L}-$ & l, L & $L, L, \perp, \Lambda$ \\
\hline$-\mathrm{M}-$ & $\mathrm{m}$ & $\mathrm{m}$ \\
\hline$-\mathrm{N}-$ & $\mathrm{n}, \mathrm{N}, 4$ & $n, n, n$ \\
\hline$-\mathrm{NN}-$ & $*$ & \\
\hline$-\mathrm{NG}-$ & 5 & $n$ \\
\hline$-\mathrm{P}-$ & $p$ & $p, \phi$ \\
\hline$-R-$ & $r$ & $r, r, R, r$ \\
\hline$-S-$ & $\mathrm{s}$ & s \\
\hline$-\mathrm{SH}-$ & $S$ & L \\
\hline$-\mathrm{T}-$ & $\mathrm{t}$ & $\mathrm{t}$ \\
\hline -TH- & 8 & $\theta, \partial$ \\
\hline$-V-$ & $\mathrm{v}$ & $\mathrm{v}$ \\
\hline$-W-$ & w & w \\
\hline$-Z-$ & $z$ & $z$ \\
\hline$-\mathrm{ZH}-$ & $z, j$ & $3, d_{3}$ \\
\hline$-7-$ & 7 & 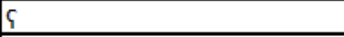 \\
\hline$-0-$ & 0 & $\odot,\rfloor, !, \neq, \|$ \\
\hline
\end{tabular}

Some of the classes are particularly challenging in the word encoding: it is not always clear if a sound has to be classified as a consonant or as a vowel. This is particularly striking within the classes "-J-", "-NN-" and "-W-". We apply clear rules to convert the source (the AJSP database 8 and ASJP sound classes [59]) to our format and there may remain room for discussion whether some sounds are correctly treated as consonant or not. However, for our methodology, the most important is to make sure that a given rule applies without exception to all datasets, without subjective human intervention. The system may codify some vowels as consonants. If it does so, it does this in a regular way, allowing these sounds to be treated according to the very same clear rules throughout the system.

\section{Pairwise comparisons}

Comparison of words across different languages is a critical task for quantifying language similarity. A common way to perform word comparison is based on the methods for sequence alignments. Sequence alignments intend to discover similarities or differences between sequences algorithmically. There have been developed different systematic pairwise alignment approaches in computer science dated back to 1970s (e.g Needleman-Wunsch, Smith-Waterman), which calculate sequence similarity first by computing the optimal alignment for two sequences and second by deriving a distance score using underlying scoring matrix for sound correspondences. We implemented our own systematic rule-based approach to determine word similarity, which is specifically adapted to the short sequences due to the fact that we compare only consonants, assessing full and partial matches. This approach of processing a pairwise comparison consists of three steps at following levels: 


\section{Syllable to syllable level (sound matching rules):}

Each syllable of the first word is compared with the syllables in the second word. Inferring syllables automatically is a task, which leads to imperfections. What the system needs is not necessarily to distinguish syllables in a strict linguistic sense but to define which the smallest unit it compares within the words is. For convenience, we call the smallest units to be processed a syllable, even if they are not always syllables stricto sensu.

The sound change model we use for matching rules in syllable to syllable comparisons consists of points from 0 , which corresponds to no resemblance to 100 for the same consonant on both sides of the syllable to syllable comparison.

To quantify differences between sets of data, a clear and stable model has to be established. Sound change quantification is the way we choose to measure erosion. Sound change is very complex and often specific to the various language families. The search for universal aspects of sound change applying in all languages necessarily leads to approximations. However, to process our comparisons, we need a set of rules, which delivers relatedness signals across all language families. We infer the rules best suited for a global classification with an iterative process we also use for the choice of lexical items, as described in §IV. We do not try to integrate as many items as possible, but want to find relevant ones with a fair degree of stability and universality. For this reason, we use only the part of sound change, which the system identifies as relevant in most language families. We need to choose the strongest signals, with the lowest possible chance interference. To do so, we apply an approach of word alignment according to our chosen rules arranging the consonants to identify regions of similarity that may be a consequence of evolutionary relationships between the words.

Our starting point of the search for a suitable sound correspondence model is the IPA $[12$ chart.

Fig 2. IPA chart for pulmonic consonants.

THE INTERNATIONAL PHONETIC ALPHABET (revised to 2015)

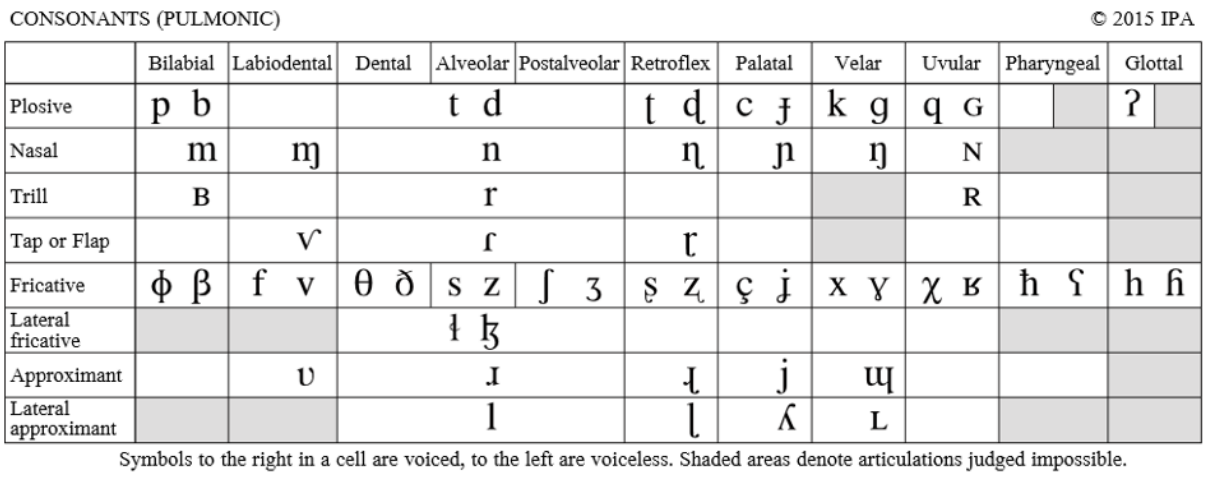

As described in $§ I$ 'Word encoding', we regroup similar consonants into classes. A match within the same class is the first, most obvious, sound correspondence rule: a same consonant or a same class at the same position on both sides of a pairwise comparison yields the maximum number of points (100). When the consonants in a same IPA cell are coded in our system in different classes (e.g. 't d', 's z', ' 3 ', ' $\mathrm{k} \mathrm{g}$ ',...) we apply a second set of sound correspondence rules with a own point level category. The third set of rules - with a further lower point level category - 
is a supposed correspondence between plosives on the one side and fricatives on the other side (e.g. 'p f', 't s', 't e' , 's e', 'd ð', 'p $\Phi$ ', 'b $\beta$ ', .. ). Although these correspondences are often observed in vocabulary comparisons across languages, they may seem highly speculative for universal comparisons. For this reason, we test their impact in the iterative process described in $\S \mathrm{IV}$, inferring a list of "best rules". We also test further rules observed in comparisons - these rules go beyond the described three categories and are compiled in a fourth one only if they appear to yield interesting results (more true positives than false positives). The outcome S2 File is a quite limited set of correspondences, which will seem familiar to anyone with a certain experience in vocabulary comparison across languages. There are hundreds of possible rules, which have a 'local' impact within and between the respective language families. Most of these rules are sorted out by the system because - on a global basis - they bring far less relatedness signals than statistical noise.

The decision, how many points each sound correspondence category yields in comparisons is arbitrary at the beginning. We have chosen 'Category 1: 100, Category 2: 90, Category 3: 75 and Category 4: 50' point model to start the analyses. This model was challenged in the iterative process described in $\S I V$ and the rounded values '100-95-85-75' have been inferred by the system as the ones, which delivers the best results. The goal is to reward the identified relatedness signals on the one side, but to differentiate them according to the erosion level on the other side.

To summarize, we can represent our sound correspondence model in following four categories:

- Category 1: same sounds (e.g. 'k - k', 't - t', 's - s', 'f - f'...) yield the maximum number of points in comparisons (100).

- Category 2: very similar sounds (same 'cell' in IPA table, e.g. 'k-g', 'p - b', ' $\mathrm{f}-\mathrm{v}$ ') yield 95 points.

- Category 3: algorithm-selected correspondences between plosives on the one side and fricatives on the other (e.g. 't - s', 't - $\theta$ ', 's - $\theta$ '...) yield 85 points.

- Category 4: other correspondences, inferred through the iterative process described in $\S \mathrm{IV}$ (e.g. ' $\int-\mathrm{s}$ ', ' 3 - z'...) yield 75 points.

Each sound change rule we apply carries a specific potential of chance interference. The number of occurrences of each consonant group in the world languages is far from constant. An example in table 1 shows how a syllable with a consonant from the "-K-" class behaves in interaction with other sounds and how it accordingly exposes to chance.

Table 1. Example of sound correspondence rule used to process the comparison (class "-K-")

\begin{tabular}{|l|l|l|l|}
\hline Consonant class & Match with & Points given & Matching probability* \\
\hline$-K-$ & $-\mathrm{K}-$ & 100 & 0.1005 \\
\hline$-K-$ & $-\mathrm{G}-$ & 95 & 0.0280 \\
\hline$-K-$ & $-\mathrm{KH}-$ & 85 & 0.0166 \\
\hline$-K-$ & $-\mathrm{H}-$ & 85 & 0.0309 \\
\hline$-K-$ & $-7-$ & 85 & 0.0240 \\
\hline$-K-$ & Other & 0 & 0.7990 \\
\hline
\end{tabular}

* The matching probability is the probability the rules apply according to the occurrence of the consonant class in the languages we use. 
In a few cases, when languages undergo a high proportion of non-universal sound evolution patterns, the system tends to overestimate the distances from their respective core-families, because it skips a higher part of the relatedness signals. A good example is Armenian, which has developed a very specific sound change [14], not reflected in our model. This is a limit to the system, which affects the stability of the results: the case of Armenian is an obvious one, but this issue affects all languages to various degrees. This has an impact on the variance of the results.

The limited set of sound correspondence rules we infer cannot be compared with more elaborated sound correspondence rules inferred by comparatists. The purposes and the scope of their use as well as the methodologies of their inference are completely different.

2. Word to word level (alignment analysis): At this level, the system has to determine which of the syllable-to-syllable matches are valid.

Due to the particularity of our system, which considers only consonants and has accordingly shorter alignments to analyze, we implement an own alignment analysis algorithm. This algorithm is much more basic than state-of-the-art methods [15] or frameworks like LingPy [16], but also gives us the flexibility to implement our own empirical rules considering partial match and filters and systematically calibrate the system towards the balance between relatedness signals and statistical noise (see $§ I V)$. Our primary goal is not the detection of cognates but the calculation of a distance value, without judgment whether this value means cognacy or not.

The system first iterates a comparison of all syllables from word 1 (word for a concept in language 1) with those of word 2 (word in language 2 for the same concept as in language 1), regardless of their respective positions within the words. All pairwise syllable comparisons are stored together with their respective positions. For example, the comparison of the second consonant in word 1 with the third in word 2 is stored as a variable 'W1C2W2C3' (Word 1 Consonant 2 vs. Word 2 Consonant 3) with the value of its match. The second step consists in counting the points for all acceptable combinations of syllable to syllable matches in the word to word context. Cross matches are not allowed (eg. 2nd consonant in word 1 matches 1 st in word 2 and 1st consonant in word 1 matches 2 nd in word 2) and too distant matches are not taken into account. The valid combinations the algorithm accepts are represented in Fig 3. All possible and accepted combinations are gathered in a list, from which the system selects the best match. Fig 3 represents how syllable to syllable matches in pairwise comparisons of words with each up to four syllables are validated. THe visualisation contains four syllables on each side, but the same rules work well with any other number of syllables between one and four, on either side. 
Fig 3. Alignment rules at word to word level: valid combinations of matches

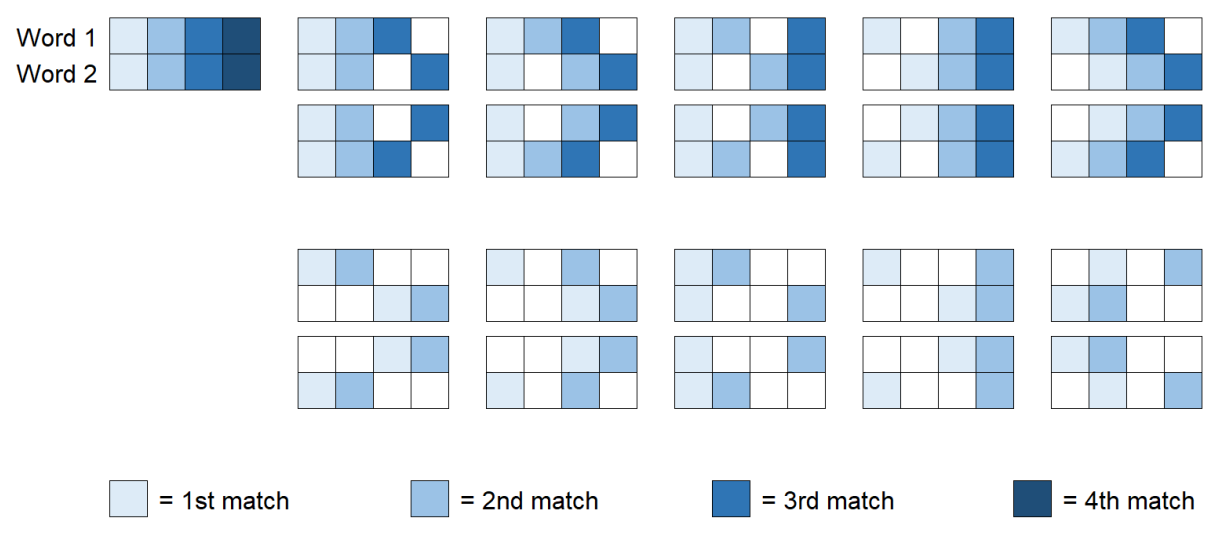

Finally, we apply a series of filters to avoid statistical noise through very low signals. Basically, if a combination of only one match other than a match on the first consonant on both sides delivers the highest score, we set this score to 0 if one of the words on either side has 3 syllables or more. The last steps consists of a filter over the result regardless of the match position: the values from which the results are filtered are inferred in a special process described in part V.

The syllable to syllable points are averaged at word level, to obtain a value from 0 to 100 for each pair of word comparison.

3. Language to language level: the points obtained at word level are averaged to obtain a value from 0 to 100 for a comparison between two languages. This value is reversed to a distance value by subtracting it from 100. The result of a pairwise comparison between two languages is a distance between 0 (same language) and 100 (no single relatedness signal, not even by chance). Specific rules eliminate points obtained with too weak signals: this step is a first filter balancing signals and noise, with the aim to eliminate as much chance matches (false positives) without losing too much real signals (true positives). We refer to it as the 'low signals filter' further in our research. This filter plays a major role in the process and has to be calibrated to contribute to the best possible balance between relatedness signal strength and statistical noise.

The system does not calculate the proximity between languages by counting the number of cognate words in a sample, but by calculating the degree of similarity the words have. In this way, the results are continuous values and make a differentiation of the degree of erosion measurable. For example, the word "water", codified ["-W-T-R-"] gets more points when compared to German "Wasser" codified ["-V-S-R-"] than to Russian "voda" ["-V-D-"]. This system does not guarantee that the proximity between two words will always follow the actual proximity between languages - English "night" ["-N-T-"], which consonants better match Italian "notte" ["-N-T-"] than German "Nacht" ["-N-KH-T-"] is an example of results' variance. The distance value at language level is 68 for English to Italian and 43 for English to German, which better reflects the degree of difference between these languages than it does with the comparison of the sole word "night". Obviously, the variance gets lower when the number of words in the comparisons grows.

Distance values are relative to each other: Distances between languages can only be interpreted in the context of their respective distances to other languages. Browsing the 
pairwise comparisons most of the time brings highly consistent results but also variations that lead to a surprising outcome. The causes are numerous and reflect important limits:

- The system assumes a constant rate of evolution, which does not correspond to the reality. Languages evolve at different speeds $[13$ in time and place.

- We compare languages in different states. Even if the lexical items in use are resistant to massive vocabulary loans, other aspects in the history of languages influence their evolution.

- Exposure to chance affects the distance values and hence influences the variance of the results.

- In short-range comparisons, the number of differences is small, giving each of the difference a very high weight, which heavily affects the variance of the short-range comparison results. If the aim is to be more accurate on short-range comparisons, more lexical items have to be used. We choose to focus on medium- and long-range classifications and our experimental research described in $\S$ III shows that more words do not necessarily lead to better medium- and long-range classifications: the choice of the best suitable lexical items is more important than the quantity we use.

\section{Choice of the lexical basis}

The Swadesh list, a standard list of basic vocabulary, is considered as the first reference for comparative linguistics based on lexical items. Since its first release with 215 words in 1950 17 it has been a constant trend to reduce the number of words, concentrating on the most stable ones, with a final version of 100 words by Swadesh himself in 1972 [18. Sergei Yakhontov has established a 35 subset 17 and more recently, the ASJP 8 project uses a 40 words list for its worldwide classification [4]. For this project, because it is our aim to quantify language relationships as far as possible in time, we have defined following criteria:

- the words should stand for concepts which were supposedly relevant to most human populations as early as 6000 to 8000 years BP,

- they have to be stable against borrowing - known borrowings have to be excluded,

- the resistance to semantic shift has to be high.

Our first goal is to find the word list, which is best adapted to classify languages. If a word list used in our system delivers a good phylogenetic classification of few but well documented languages, we can assume that it is adapted to generate reliable data for deeper analyses. The aim is not to use as many lexical items as possible, but to find the combination that delivers the best relatedness signals, with lowest possible exposure to chance. As a starting point, we use the 40 items wordlist from ASJP project [8], from which we infer a smaller but ideal items list using an iterative selection process described in $\S$ IV.

The basic vocabulary has to be resistant to influence from outside

The selected word list has a high resistance to borrowing. It resists to the influence of French in English and - to a big extent - of Arabic in Iranian and Turkic languages. Exceptions have to be identified - e.g. the influence of Indo-Aryan in Dravidian (three 
words of the list), Arabic within the Afroasiatic family and towards other language families as well as Chinese on numerous Southeast Asian languages. We exclude loan words whenever we have the information available (in our source, ASJP, part of the known loans are documented as such and we exclude them), but we cannot rule out the possibility that unidentified borrowings from very ancient times can influence the results. The exclusion of loan words is a difficult trade off as there is no satisfactory possibility to remove them with a uniform knowledge over all language families. Sources for loan words are scarce and not always reliable.

Bias caused by different level of information about loan words across language families

Removing loanwords biases the uniformity of the results' quality in a direction that favors the performance of the classifications of languages for which loan words are well-documented in the sources. We chose to accept this bias in favor of the inclusion of all available information, even is this information is not equally available over all families. The quality of the data collected in the ASJP varies from language to language, with more or less concepts missing, no or many synonyms recorded and varying focus on loan words documentation. For many languages, the sources are based on the fieldwork of one or a few researchers (e.g. Amazonas, New Guinea) or even incomplete wordlists compiled decades or centuries ago.

\section{Synonyms}

Our source, the AJSP database [8], contains synonyms. The abundance of recorded synonyms varies strongly from language to language, which is not only due to the non-homogeneous availability in the respective languages, but also to the different approaches of the various authors who gathered the data. For some languages, the source contains up to 91 entries for our 33-words list. One approach, which we tested, is to always include synonyms and retain the match with the highest result in the word-to-word comparisons. This approach also delivers a bigger exposure to chance, which in turn strongly biases the results in form of statistical noise, especially with languages for which abundant synonyms where gathered. This also biases the calculation of confidence values of the results. To avoid this - and not reject the rich source of information these synonyms carry - we chose to keep the first entry from the records, but in some cases, which we documented in the supplementary material, we selected the entry which best matches the other languages within the same subfamily (WALS genus). This in turn was done only if the change permutes an isolated form with one of the forms clearly repeatedly available in the family. This approach can influence the results in a way that favours longer-range classifications and have a negative impact on the short-range family detections. Since only one entry can be used, we chose to favour the entries which help to identify relatedness in long-range.

\section{The ideal number of lexical items}

Throughout our research, it became clearer and clearer that more words do not mean better classifications. As observed in other researches, very few words have a high degree of stability against borrowing, semantic shift and phonological erosion over a longer time frame 19. In Fig 4, we see that the variance of the distance values in language to language comparisons decreases as the number of lexical items in use increases. But we also see that this benefit become weaker, especially when we have $30+$ words in the comparison. Details on how we selected the word list and its ideal size is developed in $\S \mathrm{IV}$. 
Fig 4. Impact of the number of observed words on the result's variance (comparisons between unrelated languages)

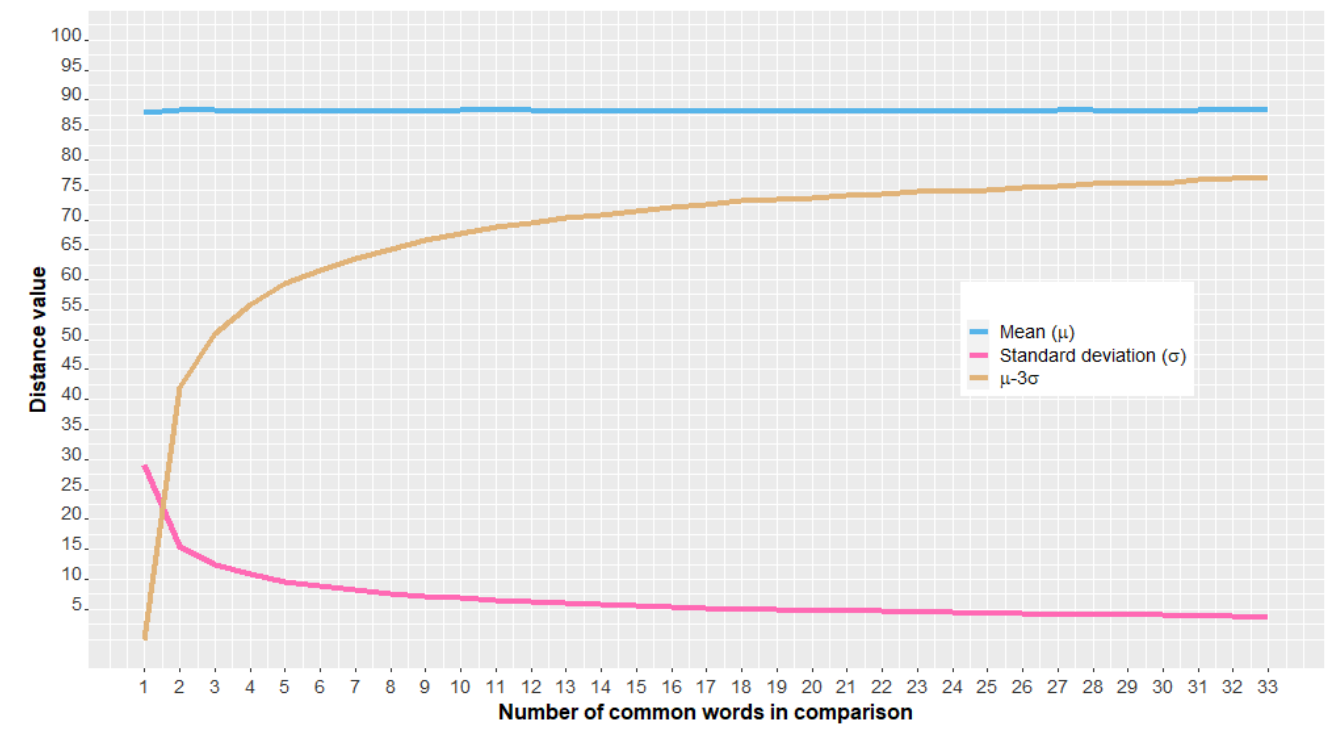

Using strict meanings vs. related meanings

The search for long-range relatedness of languages applied by various authors delivers an abundant evidence for connections between languages beyond the universally recognized macrofamilies [20] 21] 22] 23]. The longer the comparison time depth, the more indirectly signals come: relatedness in semantically shifted meanings deliver a significant part of the evidence. However, in these cases, the exposure to chance is bigger and more difficult to measure, opening the doors to critical judgments and discussions. For this reason, serious research is still rejected by a part of the linguist community 24]. To avoid this trap, even in the small list of lexical items we use in our project, we choose to let aside obvious evidence visible in semantic shift: for example in the concept of "dying" ("to die', 'death'), the stem "-M-R-T-"/"-M-R-"/"-M-T-" is present in languages across several macrofamilies. In Germanic languages however, this stem has shifted to the 'active' concept of death ("Murder"). In our system, we compare the words only from their strict meanings: automatic rules support no exception. Using this kind of evidence would mean that we include partially subjective expert judgment to our otherwise unmodified objective sources. It would also make statistical assessment of the results impossible.

All words have been converted to the format of this project (consonant classes see $\S \mathrm{I}$ ) and codified using the same criteria, excluding vowels. The conversion rules are clearly visible in the $\mathrm{R}$ script available in the online Supporting Information S3 File.

The sources and the choice of the languages to compare

We choose to apply our system on all language families reflected in the source we use: the ASJP 8 database. Many ASJP doculects share the same ISO 639-3 code (e.g. numerous Pashto, Wakhi, Romani, Shina and Hindko doculects). As we are using only consonants, the low degree of differentiation between very similar doculects makes our system very sensitive to smallest differences, including imprecisions in sources and codification. For this reason, we choose to limit the number of languages by using only one doculect, the one with the most documented concepts and synonyms (if several doculects have an equal highest number of concepts), for each ISO 639-3 code. 
In all available sources - and so in the ASJP database in use here - the sources for many languages are incomplete and make only a part of the word list available. Fig 4 makes clear that when using only few words, the variance of the distance values is much too high: the point level reached by chance increases when the number of available words for the comparison decreases. Different word availabilities in the samples bring different levels of exposure to chance, which biases the results in the phylogeny. For this reason, we choose to limit our dataset to languages, for which at least 30 words out of the 33 we use in our source (the AJSP database [8]) are available - with a few exceptions for languages which have a key position in classifications (e.g. Hittite, Gothic, Maori - 1,67\% of our sample) and for which we set the limit down to 26 words. This is crucial to limit biases in the clustering process.

\section{Search for the best set of words and sound change rules}

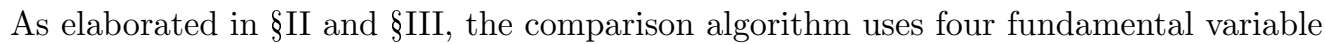
inputs:

- A lexical item list of basic vocabulary.

- A sound correspondence model.

- A point system within the sound correspondence model.

- A low signals filter in the algorithm itself (trying to act as squelch in radio transmissions).

These lists, models and numeric values are all variable. By varying the setup of items in the word list and/or sound correspondence model, as well as by trying different point assignment values and low signal filters, we produce different phylogenies, which each partially matches differently the reference classification. We do not change the input data itself, but the parameters according to which the input data is analyzed. Changing word lists and observing which words contribute to produce better results is a common practice in most lexicostatistical research. This can also apply to the other aspects - sound change rules and parameters used in the algorithm. However, this brings us to one fundamental issue. We get better results by varying parameters, but is this not the result of chance? We make multiple iterations, so we increase the chance to find the result we are looking for. Reflecting the observation "If you torture the data long enough, it will confess [25]", we can wonder if the data 'confesses' what the reality is or if it 'confesses' what we want it to confess? To avoid doubts about this issue, we choose to use a systematic method, which allows not only to infer the parameters, which generate the best possible classification from the available data, but also to make sure the good results are not simple overfitting: the best possible representation in a given context but not suited as a general model.

There are several millions possible combinations which can be applied combining the four available criteria 'Word list', 'sound correspondence list', 'point system and 'low signals filter'. If we consider alone the possibility to select 15 words out of a prefixed

corpus of 40 words, the number of possible word combinations is $\left(\begin{array}{l}40 \\ 15\end{array}\right)=40,225,345,056$. To find the best combination, we apply a heuristic optimization process, modelling a cost function, which we use to minimize an error. The system applies multiple word and sound correspondence lists to iterate a series of classifications for languages for which 
the classification is known and universally accepted. We take a subset of the classification by Glottolog [26 as our reference and use it as our 'response variable'. In a further series of iterations, we apply the same system changing the values of the point system and low signals filters. We compute phylogenies changing the lists and parameters until we reduce the error between the computerized classification and the reference classification to a minimum. We combine the search for the best word list with the search for the best sound correspondence rules and ideal parameters for the point system and low signals filter.

In this process, the system gradually excludes the items with a similarity detection power that is weaker than their exposure to chance. Whenever an item has a negative impact on the results, we can assume that it is not stable enough and/or its exposure to chance is stronger than the signals it yields towards relatedness detection. This process leads to a limited final set of words and sound change rules, as well as to an optimal point system. The minimization of the number of items is the result of the error reduction process towards a complex reference classification, which leaves very little room to chance.

\section{Overfitting}

As mentioned before, a possible limit of this approach is that computer-based algorithms are strong in modelling predictor variables towards complex classifications, but the possibility to handle thousands of 'try and error' iterations also may deliver a model, which generalizes well in the context we train it, but fails to do so in a new context. For this reason, we have split our data into two sets: a training set, which contains a part of the Indo-European, Uralic, Mongolic, Afroasiatic and North Caucasian languages and a second set, which contains all other languages. We carried out the optimization iterations with the languages from the training set, which represented $63.5 \%$ of the languages in our data at the time of the experiment. The remaining languages - for which classifications are also known and widely accepted gave us an opportunity to test our results and to see if the system also performs well for languages it has not been optimized for. The outcome was positive, although the error rates of the two categories of languages cannot be strictly compared, since their respective classifications are not equally challenging. This process has been carried out in an early stage of the project, when less than 800 languages were included in the data. Adding new languages and language families afterwards was an opportunity to confirm the system's performance in new contexts, outside the original training and testing data sets.

\section{Final selection}

The iterative process delivers optimal values for the four variable criteria of the system:

1. The best word list (Table 2).

2. The optimal set of sound correspondence items (S2 File, col. 1-2).

3. An ideal point system for 2) S2 File, col. 3).

4. The low signal filter delivering the best values - balance between reducing exposure to chance and allowing distant relatedness signals (online Supporting Information S1 File). 
Best word list

During the selection process, we identified 7 words from the ASJP 40 items list 8, which we found to have a negative impact on the reference language classification. The most negative items are "to come", "liver", "path" and "person" which negative impact was obvious in an early stage. In a second stage, the items "fish", "mountain", and "stone" were the most challenging to spot as less useful and/or negative in their impact. If we consider this list, we can find an explanation for most items: "liver" is certainly not a word suited for medium- and long-range classification, as it implies some kind of stable knowledge of anatomy at an equal level in various parts of the world. The concepts "to come", "path", "person", "mountain", and "stone" appear to be challenging in the data collection: there are often several available roots due to nuances in their meanings and it is difficult to decide which one to choose. This may explain their low contribution to classifications.

Table 2. Best word list

\begin{tabular}{|l|l|l|l|l|l|l|}
\hline blood & drink & hand & leaf & nose & sun & water \\
\hline bone & ear & hear & louse & one & tongue & we \\
\hline breast & eye & horn & name & see & tooth & you (thou) \\
\hline die & fire & I & new & skin & tree & \\
\hline dog & full & knee & night & star & two & \\
\hline
\end{tabular}

* In an early stage of the research we identified the concepts "four", "three", "wind" and "who?" as very contributive. Unfortunately, they are not available for most languages in the source we use, so we have to drop them for this research.

\section{Statistical Methods}

Our system relies on similarity signals, which are gathered from pairwise comparisons of words. When these signals are positive, this can be due to the fact that the languages are related or it can be the result of statistical noise. For example, if we take the word "to die", which is broadly covered with the consonant class chain "-M-T-" or -M-R-T-" in Indo-European languages and look for similarities outside that family, we find matches in a series of top-level families, including such for which no hypothesis exist linking them to Indo-European, like Cariban and Pama-Nyungan languages. The probability to get "-M-T-" on both sides of a comparison if the words always have two syllables is $0.074 * 0,08=0.006$ (see online Supporting Information S2 File). It means we expect to obtain such a match by chance every 167 comparison. This probability grows for partial matches (different word lengths, sound correspondences, etc.) These matches alone in Cariban and Pama-Nyungan are not a clue for relatedness. Therefore we require objective and systematic methods to measure whether observed results are significant or occur as a result of random noise.

Applying methods of descriptive and inferential statistics allowed us to make objective generalizations about pairwise comparisons for language-to-language and for middle- and long-range relationships without a manual interference. In particular, statistical inference is primarily concerned with measuring the quality of statistical parameter estimates and its variability that we obtained running the experiments. We also set up a hypothesis testing framework for the different language relationships where we consider two competing hypotheses. The null hypothesis $\left(H_{0}\right)$ that represents the perspective that two languages or language families are not related and the alternative hypothesis $\left(H_{A}\right)$ claiming that two languages or language families are related. We 
established a hypothesis testing framework selecting $H_{0}$ and $H_{A}$ before seeing the actual data and so avoiding to introduce a bias into our procedure. Our aim is to accept or to reject the null hypothesis using our data.

However hypothesis tests are not without faults and still one can lead to wrong decisions by wrongly accepting the alternative hypothesis when actually the null hypothesis is true (Type I Error) or fail to reject the null hypothesis when the alternative hypothesis is true (Type II Error). We are able to quantify the strength of the evidence against the null hypothesis by calculating the probability (p-value) of observed data or more extreme value given the null hypothesis is true. Formally the p-value is a conditional probability.

First we describe a key part of the statistical framework that will be encountered repeatedly through this paper. Since we do not know the theoretical type of the distribution that best describes unrelated languages, we construct an empirical distribution for unrelated languages by generating random languages from our data set. In this way we will be able to assess statistically whether two languages or languages families are significantly similar or not. This empirical distribution will be representing our null hypothesis $H_{0}$ or in other words, it represents the null distribution. Moreover a simulated data set of randomized languages will also enable us to visually assess the stability of derived phylogenetic tree in later stage.

\section{Null Distribution from generating randomized languages}

To simulate null distribution we first generate random words. For this purpose we first estimated several empirical probability distributions. Each lexical item is treated separately: the respective lengths are typically shorter or longer in almost all world languages and the consonant classes of a word have different probabilities to appear. Therefore, we used a probability distribution for consonant class occurrence (Fig 5) and word length (for example approx.. $48 \%$ of all words in our data have two consonants.)

Fig 5. Probability distribution of consonant classes

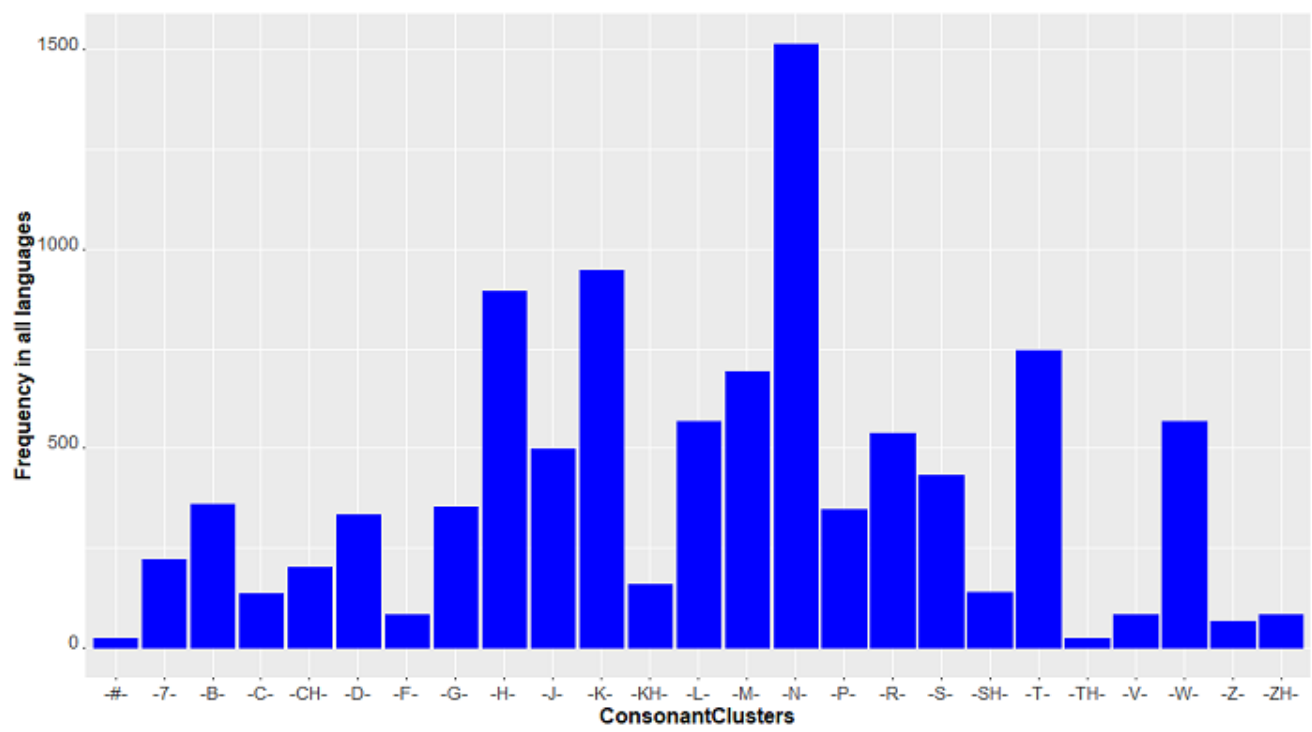

Also, we considered the fact that some consonants are more likely to occur at a particular position in a word where some other consonants are less likely to do so. 
The last issue to take into account is how often the respective words are available in our source (the AJSP database [8]). We need to replicate the lack of availability of certain words in a certain proportion in the random data we generate.

To make sure the generated random words are good representatives of the statistical noise observed in the data, we carry out statistical tests of the distributions of the comparison results between random languages against the distributions of comparisons between languages we know as unrelated. Our goal is to generate random languages, which behave the same way as real languages. This approach has the potential bias that we may not consider enough features to generate the random words. Therefore, it is sticto sensu an approximation of random languages. This method is interesting to gain understanding about how pairwise results have to be considered in the context of chance. However, using it involves processing each single pairwise comparison with a big number of random languages, which would increase the processing time of the distance matrix for all languages from 5 days to several weeks or months. Moreover, as a basis for bootstrapping, this method is not more challenging (in fact even slightly less challenging than the next ones).

\section{Null Distribution from non-related languages}

In an alternative approach, we gather the distance values of pairwise comparisons between languages from different top-level families, for which no hypothesis is available that they may be remotely related (e.g. Indo-European to Quechuan, Austronesian to Atlantic-Congo, etc.). We can use these values as pseudo randomized pairwise distance values. We can also use their empirical distributions to infer random pairwise distance values in unlimited quantities. These two related approaches also have their pitfalls: the choice of the families we use for comparisons has to be very conservative: if we compare two families, which are reported as not related in reference classifications, there is a risk that we use comparisons between families that are actually related, but this relatedness is not considered so far as proven. For this reason, we take care not to select comparisons between families, for which some hypothesis about a potential genetic relationship exists - even if this hypothesis is broadly rejected (Uralic to IE, Turkic, Mongolic, comparisons between American top-level families... ) A further source of bias is - of course - the potential presence of undetected loan words across unrelated families. Using comparisons between languages families which are geographically remote contributes to reduce this bias.

\section{Null Distribution from random sample}

Another approach to generate a null distribution when performing a hypothesis test involves taking a random sample from the data set without making any prior assumptions of the data. This approach is particularly simple to implement and when sampling the data set with replacement we even can generate a sampling distribution for any statistic of our choice e.g mean and standard error of the distribution. This is the method we use throughout our research.

\section{Language to Language}

In order to evaluate the statistical significance of similarity between two languages we estimate an empirical $p$-value based on a bootstrap simulation that samples the null distribution with replacement. Having the observed distance score $\tilde{s}$ between two languages $L 1$ and $L 2$ calculated as :

$$
\tilde{s}=100-\frac{1}{n} \sum_{i=1}^{n} d\left(L 1 W_{i}, L 2 W_{i}\right)
$$


where $n=\min (|L 1|,|L 2|)$ and $d$ being a distance function between $\operatorname{word} s_{i}$ of $L 1$ and $L 2$ respectively. Moreover we generated a vector $s=\left(s_{1}, s_{2}, \ldots, s_{N}\right)$ where $s_{i}$ contains simulated bootstrap statistics from our null distribution. Bootstrapping is a resampling technique that randomly samples data with replacement creating many simulated data. This allows to perform hypothesis testing for statistical parameters. The nature of how our distance values are determined and the fact that they are physically bounded between 0 and 100, unrelated languages having higher scores than related ones, has an impact on the shape of the distribution. In Fig. 6 we can clearly observe that the null distribution will be left skewed having related languages concentrating in the tail at the left of the distribution. For this reasons we are considering a one-sided statistical test:

- $H_{0}: \tilde{s}=\hat{s}, L 1$ and $L 2$ are not related

- $H_{A}: \tilde{s}$; $s, L 1$ and $L 2$ are related

In order to calculate the p-value we count the number of statistics $s_{i}$ that are greater than or equal to the $\tilde{s}$ value and divide by the number of the experiment size:

$$
p-\text { value }=1-\frac{\operatorname{sum}(\tilde{s}<=s)}{N}
$$

In a simplified view, setting the significance level $\alpha=0.05$ we reject the null hypotheses when $p-$ value $<\alpha$. Since we conduct multiple statistical tests for all the language pairs we make $S=(1962 X 1962) / 2=1924722$ comparisons. When performing a large number of statistical tests, some will return $p-$ values $<\alpha$ purely by chance, and may wrongly accept the alternative hypothesis causing a Type I error. To overcome this, applying a conservative Bonferroni correction can be a more appropriate approach: we correct the p-value by the number of tests being conducted, having a new rejection policy $p$-value $<\frac{\alpha}{S}$. However, in the context of results with high variances, the p-value is a help for the interpretation and certainly not a tool to firmly accept or reject an hypothesis.

Fig 6. Distribution of pairwise comparisons: IE languages vs. randomized languages

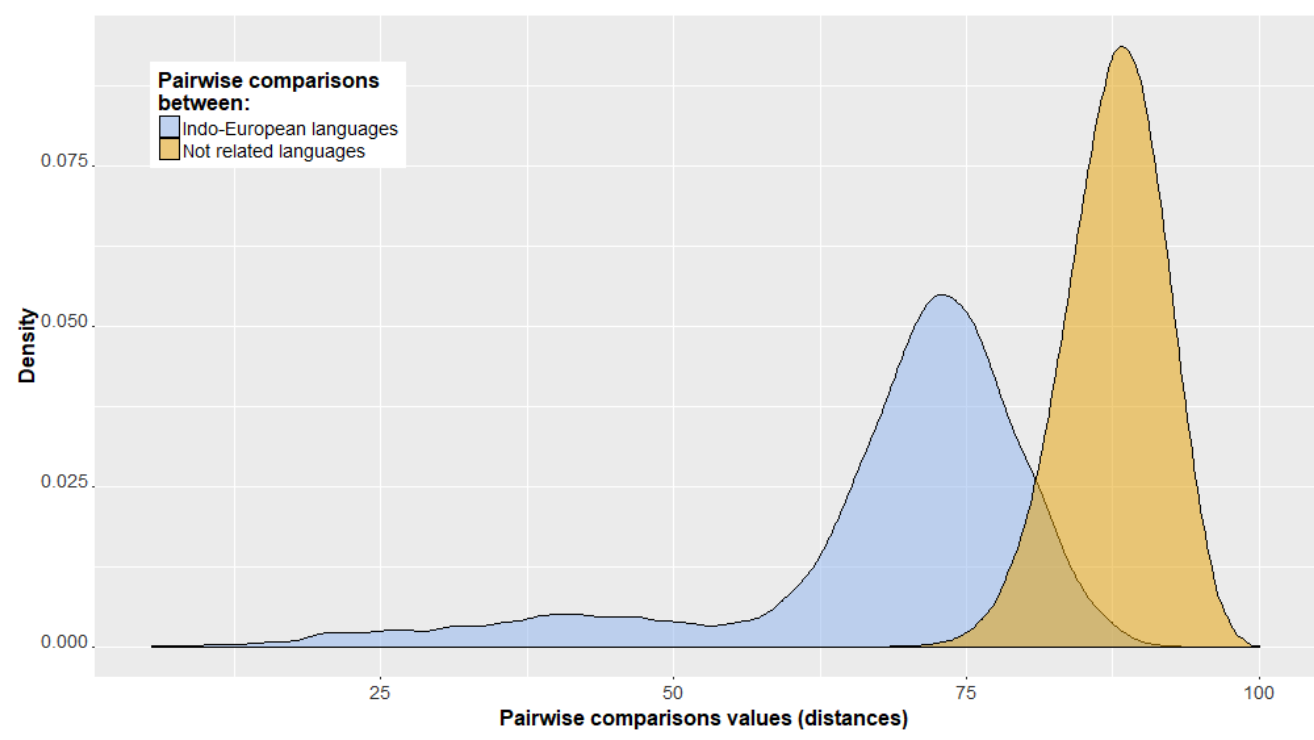


Language Subfamily to Subfamily - middle-range

To assess statistical significance between language subfamilies $(S F 1, S F 2)$ we want to test the null hypothesis that distribution of distances between all the paired languages within $S F 1$ and $S F 2$ have the same observed mean $\mu_{x}$ as the mean $\mu$ of the null distribution, however not necessarily the same distribution. In order to conduct this two-sample bootstrap test, we shift both distribution to have the same mean, since we are simulating the hypothesis that their means are, in fact, equal $\mu=\tilde{\mu}$. We then draw bootstrap samples out of the shifted arrays and compute the difference in means.

- $H_{0}: \mu_{x}-\mu=0, S F 1$ and $S F 2$ are not related

- $H_{A}: \mu_{x}<=\mu, S F 1$ and $S F 2$ are related

This constitutes a bootstrap replicate, and we generate many of them. The $p$-value is the fraction of replicates with a difference in means greater than or equal to what was observed.

\section{Language family to language family - long-range}

With the statistical framework for language to language comparisons, we generate p-values for all pairwise comparisons between languages. However, our long-range exploration is based on the analysis of relationships between top-level language families. We need a method to interpret the series of p-values generated by the comparisons between the languages of the researched families and statistically aggregate all the the p-values to make valid comparisons between top-level families. A meta-analysis of the significance values of our pairwise comparisons can be attempted using established methods to combine p-values, most of which are designed for independent experiments. In our situation, comparing the pairwise distances and their p-values for all languages from two top-level families (TLF), we have a series of comparisons which are not completely independent from each other: each TLF consists per definition of related languages. This forms a bias, which is stronger when the TLFs are more homogeneous. To reduce the problem, we do not compare all pairwise languages between the TLFs, but select only one per respective subfamily (on the basis of the WALS genus list) and always take the subfamily to subfamily comparison with the lowest distance into account. This correction helps to reduce the unnecessary inclusion of highly dependent experiments but does not resolve the dependency issue completely. A recent research by Daniel J. Wilson 27] derives a statistical technique for addressing multiple comparisons problems by combining multiple tests. It generates a harmonic mean and takes the dependency of tests into account in a meta analysis of p-values:

$$
\tilde{p}=\frac{\sum_{i=1}^{L} w_{i}}{\sum_{i=1}^{L} w_{i} / p_{i}}
$$

, where $w_{1}, \ldots, w_{L}$ are weights with $\sum_{i=1}^{L} w_{i}=1$. This approach also improves on the power of Bonferroni correction by performing combined tests. The use of weights is optional and makes sense only if information representing these weights is available in the data.

\section{Clustering - phylogenetic analysis}

One major mathematical tool to explain evolutionary relationship between languages was actually developed in computational biology and it is called phylogenetic inference, 
using a tree based data structure. In historical linguistics, the use of phylogenetic trees has expanded in the past decades [28].

There are two main groups of methods to examine phylogenetic relationships between languages:

- Phenetic methods: trees are examined by considering distance based similarities of languages, therefore require distance matrices as an input. The resulting tree is called dendrogram and it shows the hierarchical relationship between languages. It also allocates languages into clusters of highest similarity based on distances.

- Cladistic methods: trees are examined by considering various possible combinations of the tree and are based on parsimony or maximum likelihood methods. Cladistics methods do not take into account distances between languages, but rather compare characteristics of languages with each other. A characteristic of a language could be presence/absence of cognates in words. The resulting tree is called a cladogram. Cladogram show relations among languages, however it does not show how ancestors are related to descendants, even though it is possible to infer evolutionary trees from a cladogram.

Maximum likelihood methods are being considered state of the art today. They are computationally very intensive, since this method evaluates all possible trees, and calculates the likelihood for each tree using a probabilistic model. The tree with the maximum likelihood is the most probable tree.

In this research we produce a distance matrix between all possible pairs of languages. To obtain meaningful insights, we have to identify clusters of languages out of this data. A wide choice of computer programs to process phylogenies from distance matrices is available. We use the APE-Package 29] of $\mathrm{R}$ [30], which not only generates phylogenies, but also brings flexibility in analyzing the results, combining the power of a standard functionality with the ability to write own scripts for further, tailor-made analyses.

\section{Choosing the right algorithm}

The phylogenetic tools for distance based methods offer different algorithms, each of which has strengths and weaknesses. We choose to use an improved version of the neighbor joining method, BIONJ [31]. This algorithm performs better in our tests than other available distance-based algorithm: UPGMA, NJ and FastME.

When examining a phylogenetic tree it is necessary to assess the quality of the tree. We use two methods to quantify the quality of phylogenetic tree: by adding an outgroup to the tree and by bootstrapping the tree to quantify its stability. Outgroup method basically involves adding a set of randomized languages or languages being not related with other languages at all. Bootstrapping [32 [33 is a resampling technique that repeatedly samples data and performs inference about a sample from resampled data to derive statistical parameters. We analyze how well targeted references or long-range hypotheses are represented not only in the original NJS tree, but also in 100 resampled versions of it (we explain this technique in next paragraph "Results validation"). The median value of the inferred scores reflect a node support by at least $50 \%$ of the resampled trees. In practice, most of the scores are supported by far more than $50 \%$ of the trees since the result convergence is most of the time higher, with a large proportion at $100 \%$. These values can be displayed in a table only, not in a consensus tree.

\section{Results validation - Bootstrapping the distance matrix with random pairwise distances}

In a phylogenetic tree, we need to identify the stability of the inferred nodes and branches. Nodes and branches which vary when we make small changes to the 
algorithm or when we add or subtract datasets are unstable. In what is to our knowledge a own approach, we use the randomized values method (third method, "Null Distribution from random sample") and insert as many random pairwise distances to our matrices as they contain real language distance values. The insertion of different random values happens directly into 100 versions of the main distance matrix. This way, we generate 100 resampled versions of the tree (Fig 7).

\section{Fig 7. Adding random values to a distance matrix}

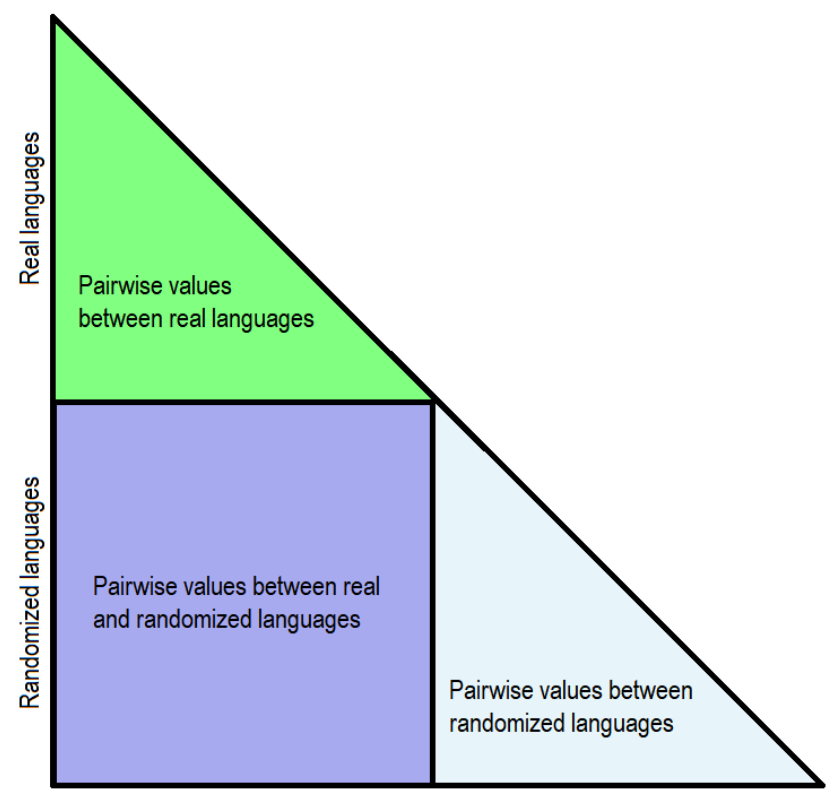

This approach enables us to infer bootstrap values from 0 to 100 . This figure reflects the number of partially randomized trees (out of 100) which support a given node. Nodes which withstand all the changes in the matrices get a bootstrap value of 100 . Nodes which get modified in 40 cases get a value of 60 , etc. How the nodes get challenged is visible if we edit a tree inferred from a bootstrapped matrix: branches with random languages as taxa mix up with real languages and form new nodes - ideally at a higher level than the nodes which make up our correct classifications. An example is represented in Fig 8.

The bootstrap values we infer are not confidence values. They are only a stability indicator in the context in which we use them. We can interpret the value for a node only when we consider it relatively to the values of the other nodes in the same tree.

To better understand how our outgroup or random values affect the trees, we represent a part of the phylogeny where random languages are included (Fig 8). The effect is the same with random pairwise values injected into the matrix or with random languages computed together with real ones before we construct the distance matrix.

This approach enables us to infer the bootstrap values of the nodes of the original NJS tree. They tell us for each node how often it is found in the same state in the 100 resampled trees. However, observing the resampled trees, we see that they often display connections (via nodes) which are not visible in the original tree. Adding random languages values to produce resampled trees has a "cleaning" effect, clustering instable signals with the random signals. To measure the value inferred by this "cleaning effect" - and thus infer bootstrap values for the nodes which are visible only in bootstrapped 
trees, we match each resampled tree with a reference and/or researched classification and get the opportunity to extract either the best values or - to reflect stability - the median values for the frequency the given node is supported in the 100 trees. This is the "Maximum Likelihood alternative" for our non sequence-data based system explain above. We show in Part II of this paper how this effect helps to improve our results and to support the search for longer-range relationships between language families.

Fig 8. Example from a raw tree: challenging Altaic connections with randomized languages

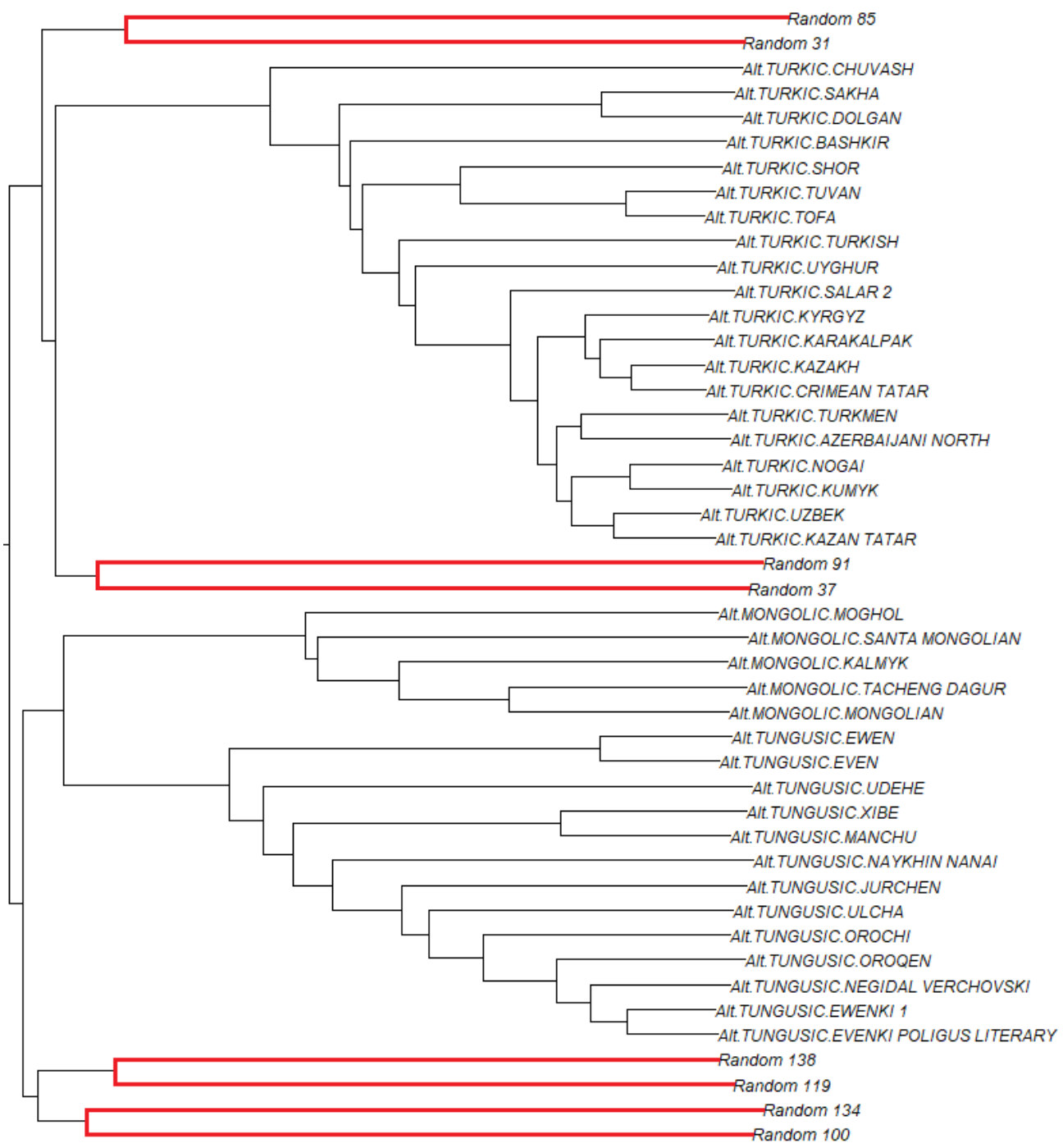

Languages for which fewer words are available

As explained in "Materials \& Methods/Choice of the lexical basis", languages for which fewer words are at our disposal, especially the ones with very few known items like Phrygian, Mycenaean, Oscan, Hurrian, Safaitic, etc. bias the classification in the phylogeny: with their very few lexical items, they do not have the same exposure to chance as languages with a complete set of meanings available. We have the possibility to include such languages (some of which are not available in the ASJP database but in 
other sources) in our data set, and to compare them with all other languages, even with randomized ones and to infer distances with corresponding p-values. This is done outside the phylogenetic analyses.

\section{Results}

\section{Pairwise results and their interpretation}

We have explained how we generate basis data for language classifications based on the consonants of a set of basic vocabulary items. To interpret the results, we have to take some time to understand them in their raw state and consider what this data is - and what it is not.

The distance values computed in single comparisons are certainly interesting for a wider audience, as people like to express any phenomenon with figures. Quantifying observations - even abstract ones - is popular. However, reducing complex phenomena to a single figure leads to approximations. With our system, each pairwise comparison of languages leads to a single value. We should neither overestimate nor underestimate it and avoid the possible confusion, which consists in considering this figure as the genetic distance value between two compared languages. The figure we infer is a distance value based on up to 33 lexical items with a certain algorithm. Of course, we have designed the choice of the items and the methods behind the distance calculations to make this data correlate as much as possible with a hypothetical genetic distance, but as with anything in nature we observe in the context of a correlation, there is a variance. If we consider a straight line between English and languages from a same subfamily, the values should all be the same - but they are not. This is due to the variance of the results.

A characteristic of a distance value in its statistical context is that it does not state whether two languages are related or not. It delivers a distance value and its p-value (in our context: the probability that the observed distance and a smaller one are due to chance). There is no judgment about relatedness. In many cases, related languages share a large distance with a very high p-value (false negatives) and unrelated languages have a relatively small distance with a very low p-value (false positives).

The distance values correlate with what we perceive as language relatedness. The question is not, whether a single result is right or wrong but how well and with which variance it correlates with language relatedness. A way to illustrate this is to consider all pairwise comparisons of English with other languages (Fig 9). If we consider these results, we see a picture, which is quite consistent with the reality we know. The nearest neighbors of English are the Germanic languages, followed by the Slavic, Roman, and other Indo-European languages. The first unrelated and random languages (red in the table) appear from distance values of about 75 and from there, they mix more and more with the most distant IE languages. From a certain value (82), no IE language appears in the list. The variance of the results is clearly visible: languages of a same language subfamily have values which are near to each other but which are not equal. When we consider the low amount of material in use, the approximations that are part of the methodology and the presence of statistical noise in quantified models based on few lexical items, we realize that the presence of a certain variance in the results is not avoidable. This variance 'contains' everything the system does not explain: non constant evolution rates, undetected loan words, not modelized complex process of evolution and of course, approximations and mistakes in gathering sources and encoding words. The more we know and take into account in our data, the lower the variance. 
Pairwise results for use in other contexts

Using distances between languages is useful for research in numerous non-linguistic fields of science (e.g. psychology, sociology,...). Language proximity can correlate with various phenomena. There is no absolute value for language distance and any attempt to establish some - like in our system - is an approximation. However, even

approximations can turn out to be useful. We publish a form for queries of all pairwise distances in the online Supporting Information S1 Appendix, which can be used in other research. The raw data is the one we use in our research. We infer also a more stable result, which averages the subfamily-wise values in pairwise comparisons to reduce the variance in medium- and long-range comparisons: for example all pairwise searches between any Germanic and any Slavic language deliver the same distance, which is the average distance between all Slavic and Germanic languages. Comparisons of languages within the same subfamily deliver the same different results as in our system. Flat files for both result sets are available in S2 Appendix 
Fig 9. Pairwise distances of English to other languages - visualization of the loose boundaries between signals and noise

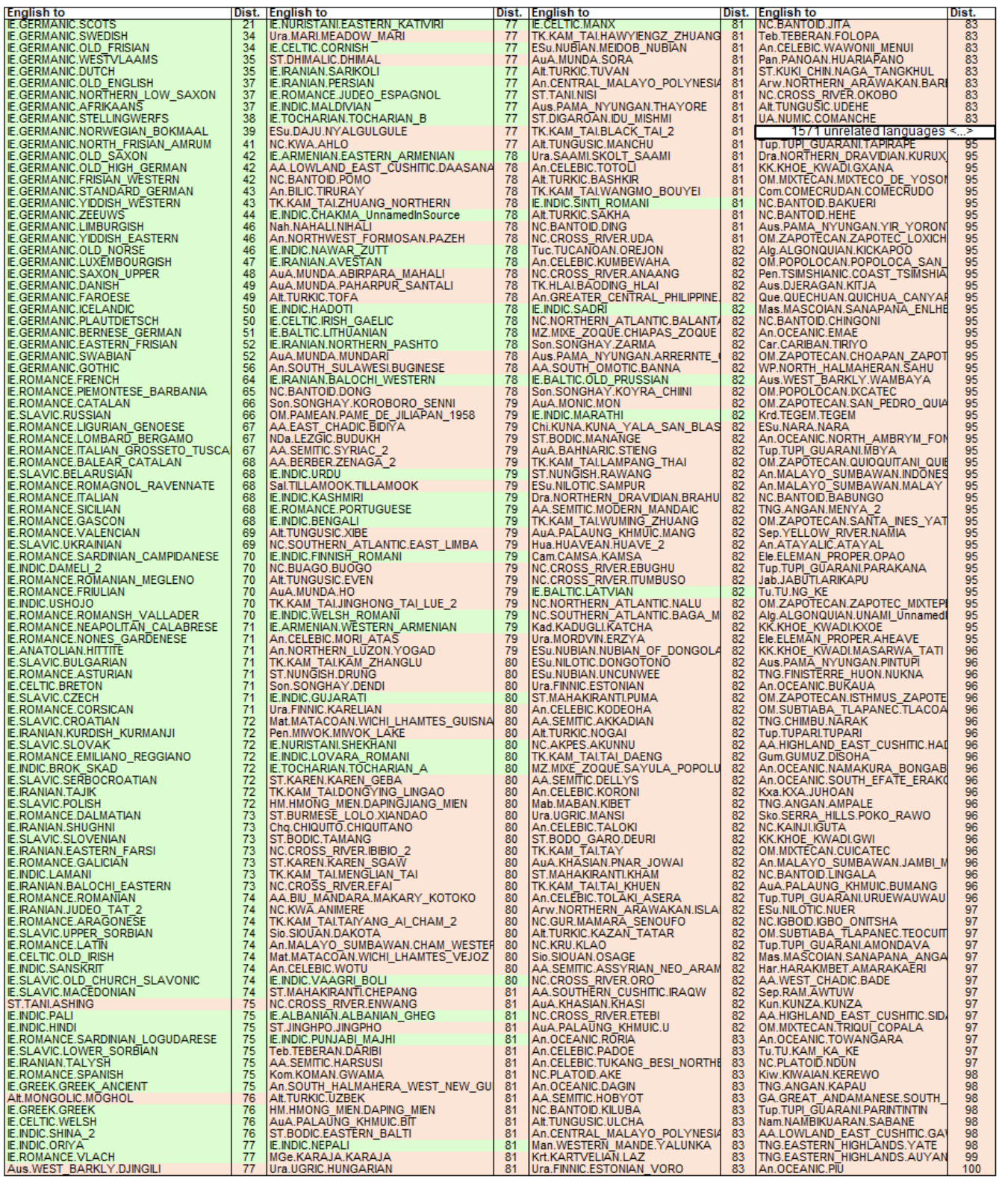

The non-averaged pairwise results come along with a p-value. In the interpretation of these p-values we have to be aware that we use more than 1.962 languages in the system. So finding a supposed distant relationship in the data, with a p-value lower than 0.01 is commonplace and not a proof for the discovery of an unknown relatedness. The p-value is a help in the interpretation, not a judgment validation. When computing multiple comparisons the odds of rare events increase, wrongly rejecting a null hypothesis.

\section{II.Inferred classifications: results and benchmark}

We deliver a worldwide classification we make available in the online Supporting Information S5 File. Fig 10 shows a subset with the Uralic languages as an example. 
Fig 10. Example of an inferred classification: Uralic

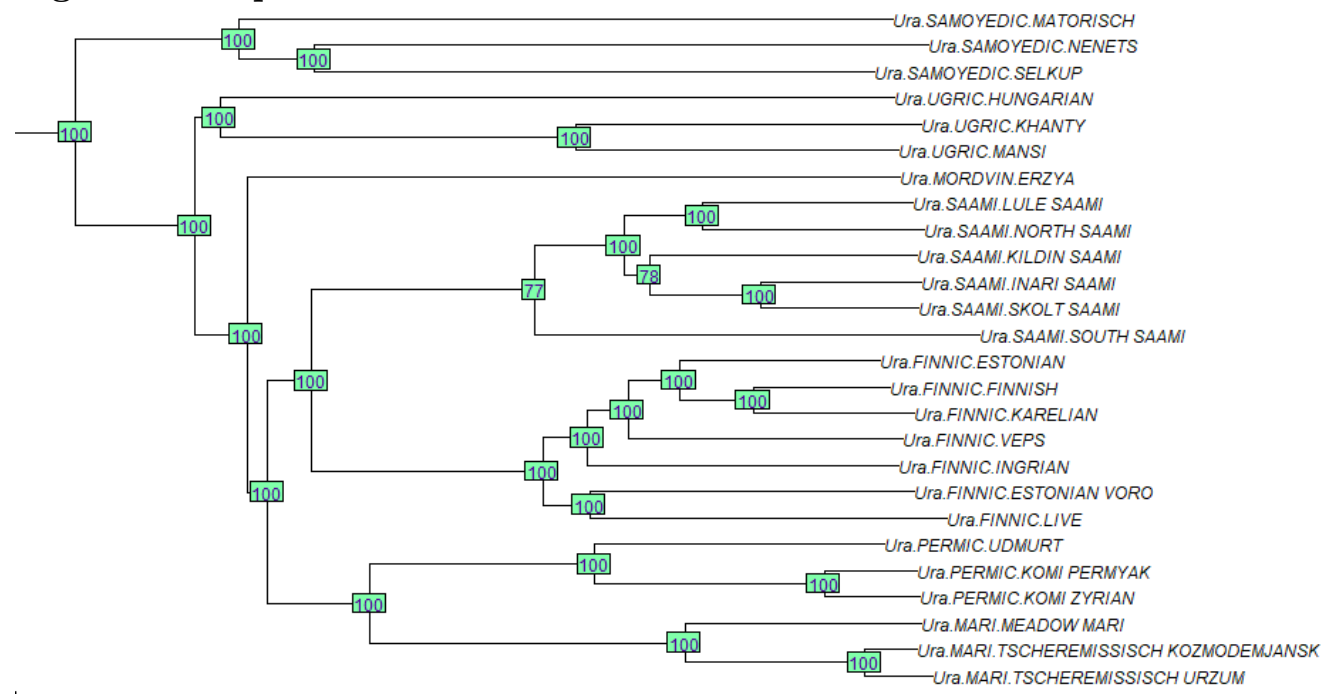

Browsing the global phylogenetic tree can be somewhat difficult to get a global overview so we also present the results in the form of a benchmark with existing classifications.

Choosing and handling reference classification to benchmark the results

We choose WALS 34], Glottolog 26] and Etnologue [35 as expert based reference classifications and Gerhard Jäger [5]'s world tree as the - to our knowledge - best computer inferred global classification available so far. Reference classifications are evolving overtime. New knowledge and research output gets included constantly. As the authors of Glottolog themselves state "the classifications are subject to change as scholarship and interest in [those] languages increases" [36]. Measuring how reference classifications differ from each other is important to make transparent what a target performance for an automatic system can be: they do not agree completely with each other, so it is impossible to reach $100 \%$ agreement with each of them: "Debate on language history is never free of disagreement among scholars" [37. An ideal automated classification will differ from the human based ones in a way, we cannot draw the difference which one is expert based and which one is computer based (similar agreement rate). We may need time to reach this level but measuring how near we are to it is a good orientation.

We use the Geiger [38] and Phangion [39] packages in $\mathrm{R}$ [30] to perform phylogenies and comparisons between/within phylogenies. Since we do not use all languages as available in all classifications, and the availability of languages differs in the different sources as well, we reduce all reference trees to the languages available in our data, common to all other sources. To make computing across several references possible, we choose to use the same language name codification as Gerhard Jäger [40]: "[WALS Family].[WALS genus].[ASJP doculect], using ISO 639-3 to link them.

\section{Measuring and benchmarking performance}

We infer three indicators to measure how well our tree matches the references:

- Score 1: a score representing the proportion of languages of a family or subfamily, which are member of the biggest cluster inferred for that family. For instance, if a 
cluster with two thirds of the languages of a family is inferred by the tree, score 1 is 0.667 for that family. If all languages are in the inferred cluster, the score is 1 . In practice, the result can split the family in several clusters. We choose to keep the value of the largest one to reflect the performance and make no difference whether the remaining languages are all gathered in a second cluster or scattered throughout the rest of the phylogeny. This value has the disadvantage that a good score for a small family (e.g. two members) has the same impact on the final result as the score of a large top-level family (TLF). So we use this score to infer Score 2, which we finally use in our assessment.

- Score 2: number of languages properly clustered in the biggest inferred cluster (Score 1 multiplied by the number of languages of the observed family). This value is derived from score 1 and eliminates the bias that big families are equally weighted as small ones in the assessment. Table 3 shows a subset of results for score 1 score 2 for a better understanding how these two scores are inferred.

- Score 3: the quartet agreement rate, derived from the quartet distance, which is defined as the number of subsets of four leaves that are not related by the same topology in the compared trees. These values, derived from the quartet divergence (Smith 2019a [41]) were calculated using the tqDist algorithm (Sand et al. 2014 [42]) implemented in the R package Quartet v1.1.0 (Smith 2019b [43]).

We have developed scripts, which allow to compare how classifications match each other and hence to measure the performance of a given classification against a reference. Table 3 shows a subset of results for score 1 and score 2 . We implement the quartet agreement rate only for top-level families with at least 10 members, as the method delivers better results for the numerous smaller trees and bias the score wrongly positively.

Table 3. Example (subset) of benchmark of our results with Glottolog

\begin{tabular}{|l|l|l|l|}
\hline Family & Size & Score 1 & Score 2 \\
\hline Indo-European & 135 & 1 & 135 \\
\hline Eastern Baltic & 2 & 1 & 2 \\
\hline Slavic & 14 & 1 & 14 \\
\hline East Slavic & 3 & 0.667 & 2 \\
\hline South Slavic & 6 & 0.5 & 3 \\
\hline Eastern South Slavic & 3 & 0.667 & 2 \\
\hline Macedo-Bulgarian & 2 & 1 & 2 \\
\hline Western South Slavic & 3 & 1 & 3 \\
\hline Serbian-Croatian-Bosnian & 2 & 1 & 2 \\
\hline West Slavic & 5 & 0.6 & 3 \\
\hline Czech-Slovak & 2 & 1 & 2 \\
\hline Sorbian & 2 & 1 & 2 \\
\hline Insular Celtic & 7 & 1 & 7 \\
\hline Brythonic & 3 & 1 & 3 \\
\hline Southwestern Brythonic & 2 & 1 & 2 \\
\hline Goidelic & 4 & 1 & 4 \\
\hline Total & 212 & 15.4 & 203 \\
\hline Percentage & $100 \%$ & $90.7 \%$ & $96.7 \%$ \\
\hline
\end{tabular}

Table 4 gives an overview how Glottolog and Ethnologue match with each other and with the WALS classification, as well as how Jäger [5]'s NJS tree and our own NJS 
results match with WALS, Glottolog and Ethnologue. These reference classifications have different granularities: the WALS classification delivers the top-level families, as well as a first level of subfamilies ("WALS Genus"). Glottolog and Ethnologue also have these levels of classification but also deeper ones. As mentioned above, these results we display are based exclusively on languages, which are common to all these classifications. The detail results are available in the online Supporting Information S4 File, The reference electronic version (Nexus) of the trees for WALS and Glottolog are taken from Jäger, G. [40. The reference Nexus tree for Ethnologue is from Dediu, D. 44

Table 4. Benchmark between classifications ('Score 2' clustered languages)

\begin{tabular}{|c|c|c|c|c|}
\hline & \begin{tabular}{l|l|l} 
WALS & 34 & TLF \\
\end{tabular} & WALS Genus & Glottolog 26 & Ethnologue 35 \\
\hline Glottolog & $93.1 \%$ & $96.5 \%$ & $100 \%$ & $84.9 \%$ \\
\hline Ethnologue & $91.8 \%$ & $95.2 \%$ & $82.9 \%$ & $100 \%$ \\
\hline Jäger's NJS tree & $82.8 \%$ & $84.7 \%$ & $61.8 \%$ & $70.4 \%$ \\
\hline Our NJS tree & $78.0 \%$ & $81.5 \%$ & $59.3 \%$ & $67.4 \%$ \\
\hline
\end{tabular}

Table 5. Benchmark between classifications (weighted agreement rates)

\begin{tabular}{|c|c|c|c|}
\hline & \begin{tabular}{l|l|l} 
WALS & 34 & TLF \\
\end{tabular} & Glottolog 26 & Ethnologue 35 \\
\hline Glottolog 26 & 0.993 & 1 & 0.868 \\
\hline Ethnologue 35 & 0.869 & 0.868 & 1 \\
\hline Jäger 5 's N JS tree & 0.635 & 0.634 & 0.630 \\
\hline Our NJS tree & 0.631 & 0.629 & 0.624 \\
\hline
\end{tabular}

The high granularity of the Glottolog and Ethnologue classifications leaves room for discussions on some families. This explains the lower agreement rate between these two families, at least on their whole scope: their respective agreement rates on less granular WALS TLF and WALS Genus is much higher.

Results and time depth - limit of tree-like patterns reflected in performance

In our and Jäger's results, the performance of the computer-inferred classifications gets lower when the time range of the classifications gets shorter (Glottolog/Ethnologue, containing classifications within subfamilies, versus WALS containing only TLF and subfamily classifications). The biggest differences are found in the subfamilies. One of the reasons lies in the limitation of the tree-like patterns, which is a practical, but oversimplified mean of representing language evolution [45]. The biases the tree model carries is much higher in the short-range than in medium- and long-range classifications. 
Our results performance represented on world maps

Our results' performance is not equal in all world areas: the performance is better in well documented language families, Eurasia delivering more stable results (Fig 11). This trend is visible more in details in the result table in the online Supporting Information S4 File. The fact that our system - but also other computer-based ones - best matches well documented families like Indo-European or Uralic shows that the major impact on results is data-quality. Data quality has a high impact on computer based classifications' performance.

Fig 11. Our results' performance (WALS genus contains all its members) on the world map

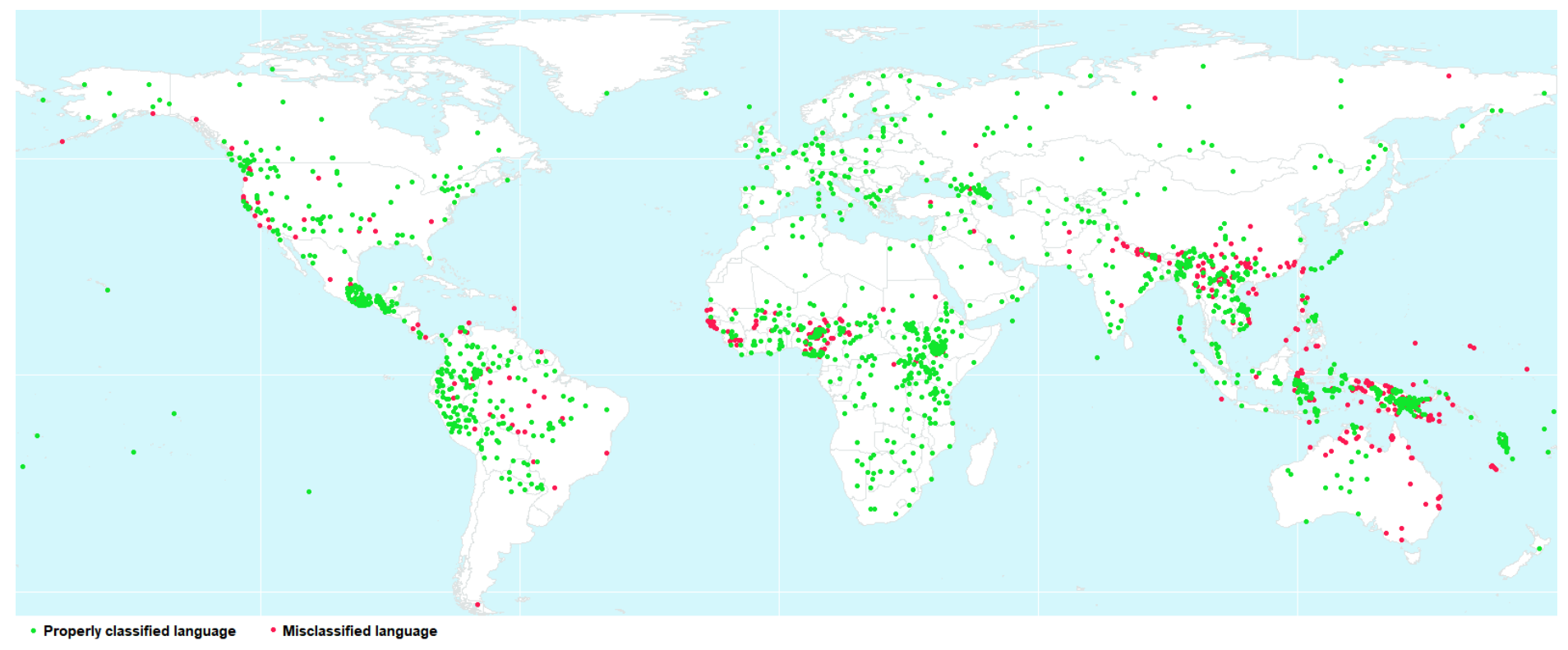

Visualizing and exploring results

The results in the form of one phylogenetic tree with 1962 languages, as inferred in our supporting information S5 File-is difficult to use in practice. The search for interesting findings is more accessible if we limit the representation to medium- and long-range results. As explained, our methodology is not designed for short-range classification and the variance of the results has its biggest impact here. The global tree contains all languages and inferred short-, medium- and long-range connections and is the reference when it comes to check the detail results. However targeting result exploration to medium- and long-range needs more proceedings. We address both in the next two sections.

\section{Subfamilies: Exploring the internal classification of top-level language families}

The inferred internal classifications of all top-level families with at least 3 subfamilies is available in the supporting information S6 File. In Fig 12 and 13, we display the internal classifications of the Indo-European and Afroasiatic top-level families. 
Interpreting the results

Among scholars, the internal classification of subfamilies leads to much more agreement about the content of the clusters (which language belongs to which subfamily) than in how these subfamilies classify within the top-level family trees (the order in which they split). The reference classifications let the subfamilies connect directly to the root whenever the consensus among scholars about specific internal splits is low. Hypotheses about how subfamilies link with each other are numerous and there is no complete agreement even for well-documented families.

Fig 12. The internal classification and node stability of Indo-European as inferred by our system vs. conservative consensus

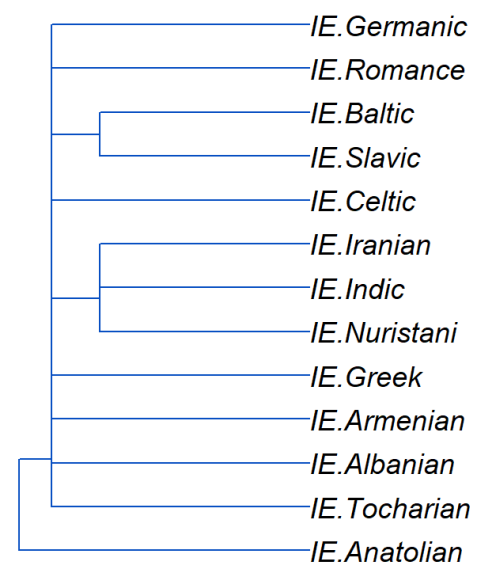

- IE Internal classification Glottolog -

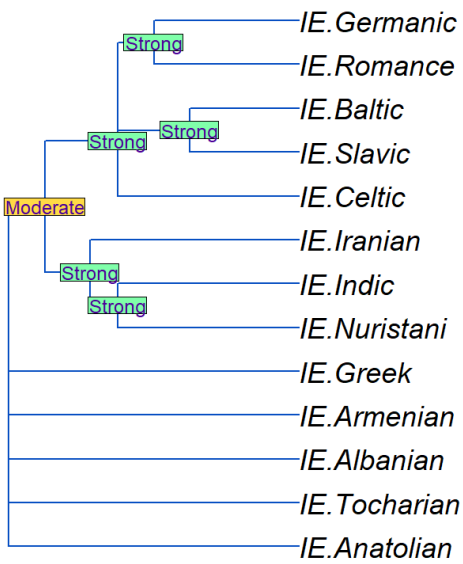

- Our IE Internal classification -

For Indo-European, the reference classifications [26 35] record only two connected sub-groups: Baltic with Slavic and Indo-Aryan with Iranian, connecting these two and all other subfamilies directly to the root of Indo-European. We know that the Indo-European subfamilies did not split in separate directions at once. In which order the splits happened is subject to debate since almost 200 years [46]. This debate is of great relevance when it comes to linking linguistic data with findings from genetics and archeology. Analyzing our Indo-European tree of subfamilies (Fig 12), we get an quantitative answer to this question: Romance, Germanic, Celtic and Balto-Slavic form a cluster. As already widely accepted, Indo-Aryan, Iranian and Nuristani form a second cluster. These two clusters, form a next level group and Anatolian, Tocharian, Armenian, Albanian and Greek are outliers. Our data suggests that Anatolian and Tocharian separated first and the rest of the Indo-European family formed a common stock. The way Albanian, Armenian and Greek separated is not visible in our data but they did so before the next stock separated in two directions: Indo-Iranian and Italic-Germanic-Balto-Slavic and Celtic with the further splits leading to the subfamilies as we know them today. If we match these results with the raw tree with all languages (supporting information S5 File the position of the IE Subfamilies with few elements (Anatolian, Tocharian, Greek, Armenian) slightly differs. Keeping the common, stable results across the two methods confirms the two big clusters

"Iranian-Indo-Aryan-Nuristani" on the one side and

"Celtic-Romance-Germanic-Balto-Slavic" on the other side.

The Afroasiatic tree is a similar challenge. Our system delivers results (Fig 13) which support existing views 47]. Dizoid and Omotic form a distant subgroup, with 
strong bootstrap support. Chadic is positioned in a long-range subgroup together with Semitic and Berber. South Omotic, which is considered as a own top-level family by Glottolog is an outgroup in our tree and is not directly linked to North Omotic. The strong link between East-Chadic and Berber is certainly a misclassification in our tree.

Fig 13. The internal classification of Afroasiatic as inferred by our system

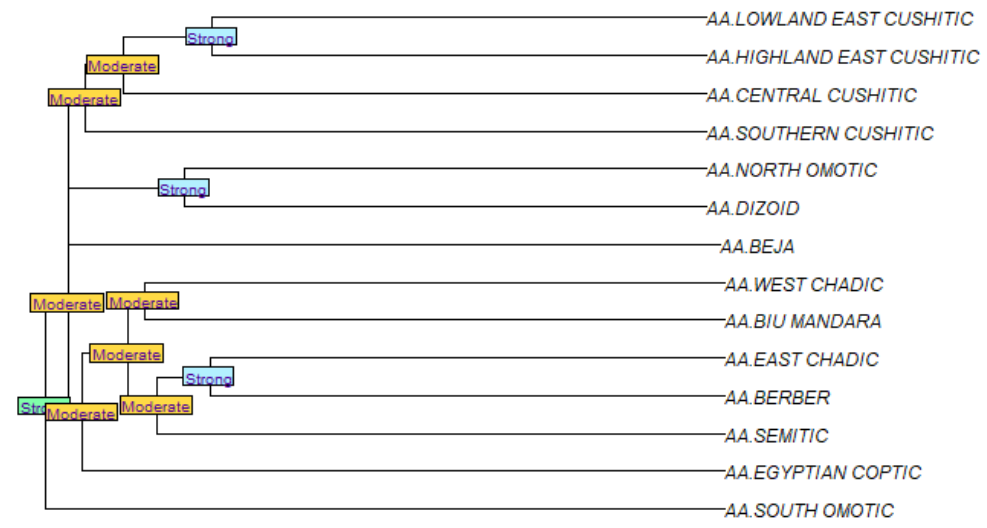

This analysis starts with the WALS Genus classification as constraint. This means that if some WALS subfamilies are regarded as disputed, a separate analysis with the diverging subfamily hypotheses should be carried out. The "strong" and "moderate" stati of the nodes refer to the bootstrap support.

\section{Exploring long-range and disputed classifications}

Exploring long-range language relationships out of the darkness of prehistoric times is a difficult path: the fascination it awakes should not overshadow the necessity to be very cautious when it comes to interpret signals inferred by various research. Reference classifications are very conservative when it comes to including new findings. It is their role to reflect results supported only by strong evidence and a wide consensus among the comparative linguists. In some cases, results diverge between the three references. The WALS classification includes top-level families, which are not supported by the other two references (Altaic, Hokan, Oregon Coast, Penutian). There is an abundance of hypotheses linking language families still pending recognition by reference classifications, even after decades of research. Some are regarded as highly speculative, other already have a broad support but still fail to meet the criteria needed for their inclusion. There are two main reasons for the doubts:

1. The lack of proof in form of regular sound correspondences and proto-languages reconstructions, which are the established methods to identify language families. With deeper time ranges, these methods become more difficult to apply, as the underlying data loses the signals with increasing erosion. 
2. The identification of weaker but "visually" convincing signals makes out the bulk of supposed evidence for long-range connections. Such signals are controversial, because of the high probability that this evidence is simply due to chance.

Our system addresses the second issue and aims at using both signals and noise (chance resemblance) as the basis of the analysis, instead of focussing on the signals alone. We do so in two different ways: analysis of the phylogenies and meta analysis of p-values, which we explained in "Materials and methods" $\S \mathrm{V}$ and $\S \mathrm{VI}$.

\section{The signals and the noise in the phylogenies}

Relatedness signals between macrofamilies are visible in the trees. However, the clues for long-range connections we get from raw trees have to be interpreted with great caution: in a rooted phylogeny, any top-level family necessarily links to some other -

the branches have to find a place in the tree, even when the values linking them have to do with chance and not with relatedness. Using the bootstrapping technique explained earlier, we can track the stability of branches. If we consider only connections between top-level families, which the system infers in a block in our world tree (online Supporting Information S5 File we find a very limited amount of stable TLF to TLF connections. We list them in table 6 . Interestingly, all of them but one are part of existing long-range hypotheses, not yet accepted by the reference classifications.

Table 6. TLF pairs' phylogenetic support (worldwide tree see online Supp. Inf. S5 File)

\begin{tabular}{|c|c|c|c|}
\hline TLF 1 & TLF 2 & Hypothesis & Bootstrap value \\
\hline Quechuan (Que) & Aymaran (Aym) & Quechumaran [48 $4 \overline{49}$ & 100 \\
\hline Tacanan (Tac) & Panoan (Pan) & Pano-Tacanan 50 & 100 \\
\hline Indo-European (IE.) & Altaic (Alt) & Eurasiatic $[18$ & 100 \\
\hline Altaic (Alt) & Uralic (Ura) & Eurasiatic $[\overline{18}$ & 100 \\
\hline Austroasiatic $(\mathrm{AuA})$ & Hmong-Mien (HM.) & Yangzian (Austric) 51 & 100 \\
\hline Matacoan (Mat) & Guaicuruan (Gcu) & Mataco-Guaicuru 52 & 100 \\
\hline Totonacan (Tot) & Mixe-Zoque (MZ.) & Totozoquean $[53$ & 50 \\
\hline Indo-European (IE.) & Uralic (Ura) & Eurasiatic 18 & 100 \\
\hline West Papuan (WP.) & Timor-Alor-Pantar (TAP) & No & 100 \\
\hline Nakh-Daghestanian (NDa) & Northwest Caucasian (NWC) & North Caucasian 54 & 6 \\
\hline
\end{tabular}

The signals and the noise in the meta analysis of p-values: Pairwise TLF results and their clusters: We apply harmonic mean p-value analysis to all possible pairwise comparisons between WALS top-level families and display the results (14368 pairwise TLF results excluding the results between the same TLFs) in the online Supporting Information S7 File. The use of harmonic mean p-values leaves only 16 observations out of 14368 with $\mathrm{p}<0.0001$. With the table in in the online Supporting Information $S 7$ File, we reach the core of the analysis of our results in the context of signals and noise. The table delivers connections between TLF with low p-values, most of which are clearly due to chance. How do we differentiate signal and noise? The four results with extremely low p-values are very clear. The other lower results are a mixture of members of existing hypotheses and members of completely unrelated connections. When we analyze hypotheses, which consist of only one pair of TLF, the differentiation between signals and noise is not possible. The contemplation of a pairwise result in the context of neighbouring unrelated results can be useful to evaluate how low a TLF pair is, for which a hypothesis exists.

When we consider clusters of more than 2 TLFs, we become far less signals, but they become easier to differentiate from noise. We inferred all the 3- and 4-members clusters 
with TLF to TLF connections supported by $\mathrm{p}<0.002$ and displayed them in the "Long-range clusters" tab of the online Supporting Information S7 File. Out of 791,489 potential clusters with three members, only 45 fulfill this condition. Out of these 45 clusters, we can form only 5 four-members clusters with all mutual connections with $\mathrm{p}$ $<0.002$. Another list of 6 clusters with more than 3 members can be listed from the 45 three-members cluster list, if we accept the p-values of their remaining connections with $p>0.002$. Ranking these 11 clusters by their p-values, we get two Nostratic and one Austric clusters in the top 5 list (details in the online Supporting Information S7 File, "Long-range clusters" tab).

\section{Analyzing results:}

In the pairwise TLF to TLF results of the online Supporting Information S7 File, four TLF relationships outside the WALS reference deliver a very clear result: Quechuan to Aymaran, Panoan to Tacanan, Mande to Niger-Congo and Indo-European to Altaic, all of which are part of existing long-range hypotheses. The other long-range hypotheses we address are among the pairwise TLF comparisons with the lowest harmonic mean p-values, but share their positions with clearly unrelated TLF pairs. This does leave us within the range of "the signals and the noise" dilemma but delivers at least strong clues, which can be combined with other facts in the discussion. We analyze some of the long-range hypotheses our system unveils both in the bootstrap analysis of the inferred phylogeny and the harmonic mean p-values analysis.

Eurasiatic [19] and Nostratic [17] [18] [20]

Eurasiatic and Nostratic form a fascinating but also highly controversial hypothesis. As a member of Nostratic, Eurasiatic typically includes Indo-European, Uralic, Altaic, Eskimo-Aleut and Chukotko-Kamchatkan, with variations by different authors.

Nostratic is a wider family, also with variations by various authors, with Eurasiatic, Dravidian, Kartvelian, Koreanic, Japonic and Afroasiatic as core members. Our system has a very clear support both in the phylogeny/bootstrap and in the p-value analysis for a core part of Eurasiatic: Indo-European, Uralic and Altaic proper (Turkic, Mongolic, Tungusic). No signal is visible for an inclusion of Eskimo-Aleut and Chukotko-Kamchatkan in Eurasiatic. Koreanic and Japonic, which are included in Altaic, Eurasiatic or Nostratic by some authors do not display any signal in this direction in our system. Beside the clear support for a core version of Eurasiatic, the p-value analysis supports two other members of the Nostratic family: Afroasiatic and Kartvelian, the later also with a noisy support (bootstrap value 44) in the phylogeny. Fig.14 shows the p-value analysis values for these connections. 
Table 7. TLF pairs' support by HM p-values (complete list see online Supp. Inf. S7 File)

\begin{tabular}{|c|c|c|c|c|}
\hline TLF 1 & TLF 2 & Hypothesis & HM p-value & Rank \\
\hline Quechuan (Que) & Aymaran (Aym) & $\begin{array}{lll}\text { Quechumaran } & 48 & 49\end{array}$ & $<0.000001$ & $1 / 14368$ \\
\hline Tacanan (Tac) & Panoan (Pan) & Pano-Tacanan $\overline{50}$ & $<0.000001$ & $2 / 14368$ \\
\hline Indo-European (IE.) & Altaic (Alt) & Eurasiatic (Nostratic) [18] & $<0.000001$ & $3 / 14368$ \\
\hline Niger-Congo (NC.) & Mande (Man) & Blench, Dwyer. Vydrin 55 & $<0.000001$ & $4 / 14368$ \\
\hline West Bomberai (WBm) & Trans-New Guinea (TNG) & Usher (2020) 56 & 0.000022 & $7 / 14368$ \\
\hline Mayan (May) & Mixe-Zoque (MZ.) & Mora-Marín (2005) & 0.000106 & $14 / 14368$ \\
\hline Salishan (Sal) & Iroquoian (Iro) & Almosan-Keresiouan [57] & 0.000127 & $19 / 14368$ \\
\hline Austronesian (An.) & Tai-Kadai (TK.) & Austro-Tai (Austric) & 0.000157 & $24 / 14368$ \\
\hline Austroasiatic $(\mathrm{AuA})$ & Austronesian (An.) & Austric [51] & 0.000186 & $25 / 14368$ \\
\hline Tai-Kadai (TK.) & Hmong-Mien (HM.) & Austric 51$]$ & 0.000349 & $41 / 14368$ \\
\hline Afroasiatic (AA.) & Uralic (Ura) & Nostratic 20 & 0.000355 & $42 / 14368$ \\
\hline Altaic (Alt) & Kartvelian (Krt) & Nostratic $\overline{20}$ & 0.000389 & $47 / 14368$ \\
\hline Wakashan (Wak) & Salishan (Sal) & Almosan-Keresiouan [57] & 0.000468 & $55 / 14368$ \\
\hline Afroasiatic (AA.) & Altaic (Alt) & Nostratic 20 & 0.000492 & $57 / 14368$ \\
\hline West Bomberai (WBm) & Timor-Alor-Pantar (TAP) & Usher $(2020)[\overline{57}$ & 0.000497 & $58 / 14368$ \\
\hline Altaic (Alt) & Uralic (Ura) & Eurasiatic (Nostratic) 18 & 0.000506 & $59 / 14368$ \\
\hline Austroasiatic $(\mathrm{AuA})$ & Hmong-Mien (HM.) & Yangzian (Austric) 51 & 0.000553 & $65 / 14368$ \\
\hline Matacoan (Mat) & Guaicuruan $(\mathrm{Gcu})$ & Mataco-Guaicuru $5 \overline{2}$ & 0.000554 & $66 / 14368$ \\
\hline Trans-New Guinea (TNG) & Timor-Alor-Pantar (TAP) & Usher (2020) 57 & 0.000619 & $72 / 14368$ \\
\hline Totonacan (Tot) & Mixe-Zoque (MZ.) & Totozoquean $[\overline{53}]$ & 0.000813 & $89 / 14368$ \\
\hline Algonquian (Alg) & Salishan (Sal) & Almosan-Keresiouan 57 & 0.000828 & $90 / 14368$ \\
\hline Indo-European (IE.) & Uralic (Ura) & Eurasiatic (Nostratic) $1 \overline{1}$ & 0.000846 & $92 / 14368$ \\
\hline Indo-European (IE.) & Afroasiatic (AA.) & Nostratic 20 & 0.001144 & $116 / 14368$ \\
\hline Wakashan (Wak) & Iroquoian (Iro) & Almosan-Keresiouan [57] & 0.001179 & $118 / 14368$ \\
\hline Afroasiatic (AA.) & Kartvelian (Krt) & Nostratic 20 & 0.001268 & $125 / 14368$ \\
\hline Algonquian (Alg) & Iroquoian (Iro) & Almosan-Keresiouan 57] & 0.001558 & $146 / 14368$ \\
\hline Austroasiatic $(\mathrm{AuA})$ & Tai-Kadai (TK.) & Austric [51] & 0.001559 & $147 / 14368$ \\
\hline Kartvelian (Krt) & Dravidian (Dra) & Nostratic 20 & 0.002094 & $183 / 14368$ \\
\hline Nakh-Daghestanian (NDa) & Northwest Caucasian (NWC) & North Caucasian 54 & 0.0024 & $211 / 14368$ \\
\hline Indo-European (IE.) & Kartvelian (Krt) & $\begin{array}{ll}\text { Nostratic } & 20\end{array}$ & 0.002446 & $221 / 14368$ \\
\hline
\end{tabular}

Fig 14. Eurasiatic and Nostratic clusters in the meta-analysis of p-values

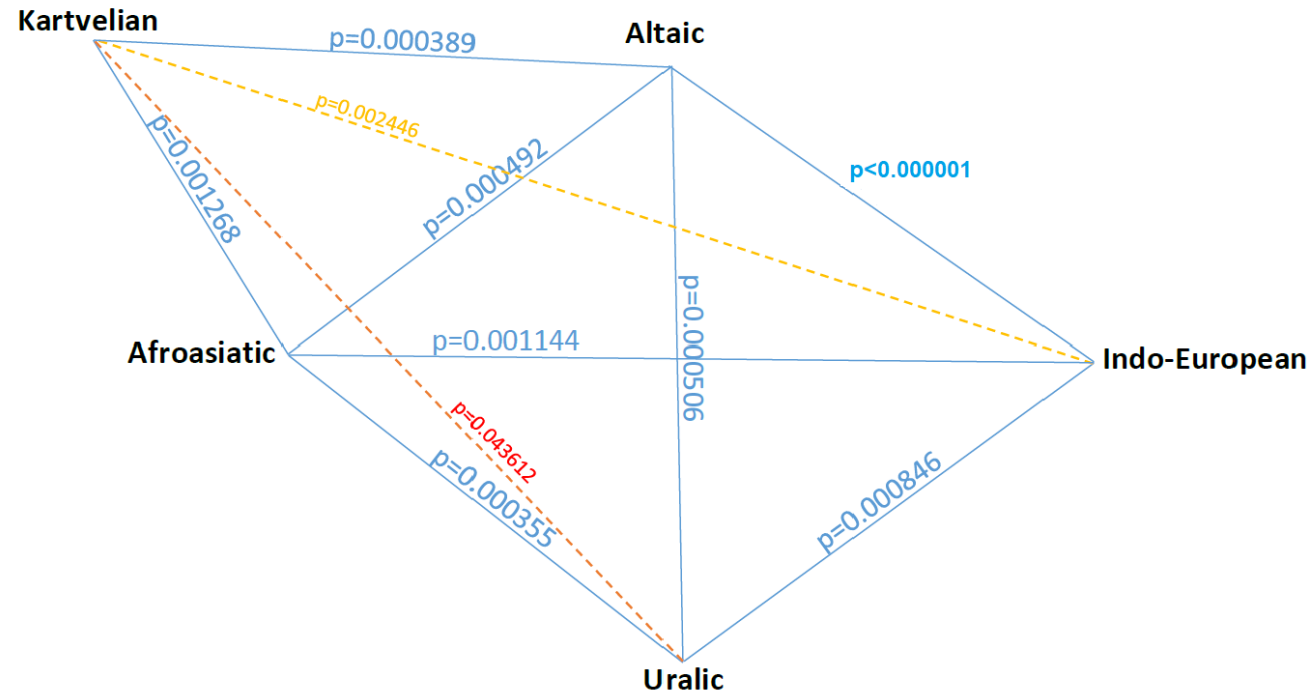


Austro-Tai, Sino-Austronesian, Yangzian [51] [58]

Our data shows a clear cluster with a proximity between the Austronesian, Tai-Kadai, Austroasiatic, Hmong-Mien and Sino-Tibetan top-level families. Austronesian and Tai-Kadai form a sub-branch, Austroasiatic-Hmong-Mien an other one, with Sino-Tibetic close to it (especially to Hmong-Mien). Since 1906 with Wilhelm Schmidt and 1942 Paul K. Benedict, various hypotheses have linked some or all of these families, sometimes with extensions to other ones. These hypotheses are known under the name "Austric". A sub-branch of Austric, Austro-Tai, linking Austronesian and Tai-Kadai show a proximity in the phylogeny and a strong support by the p-value analysis (Fig 15). Such a connection is mentioned by numerous authors, with more recent works in 2004-2006: Ostapirat (2005), Sagart (2004) and Reid (2006) supporting them. The Austroasiatic-Hmong-Mien connection is also well supported in our data, both in the phylogeny (bootstrap value: 100) and in the p-value analysis. Stanley Starosta's 58 Sino-Tibetan-Yangzian family corresponds to our cluster

"Austronesian-Hmong-Mien-Sino-Tibetan" and our data also hints at his East Asian hypothesis, connecting Austro-Tai and Sino-Tibetan-Yangzian. Our Austro-Tai and Yangzian connections also fit as parts of Sergei Starostin's Dene-Daic macrofamily but the position of Sino-Tibetan is in this case too close to Yangzian. This proximity as inferred in our system is due to a Sino-Tibetan-Hmong-Mien-Japanese cluster which is probably due to early language contacts. Japanese and Chinese have a low HM-p-value support to the members of the Austric cluster other than Hmong-Mien, whereas within the Austric cluster, the mutual support is stronger. This can be interpreted as the signal of a differenciation between macrofamily (Austronesian, Austroasiatic, Tai-Kadai, Hmong-Mien) and macro-area (the latter with Chinese and Japanese). However, even with these results, this interpretation should be made with great care, as early contact within the ancestor languages of the Austric group cannot be ruled out.

Fig 15. Larger Southeast Asian clusters in the meta-analysis of p-values

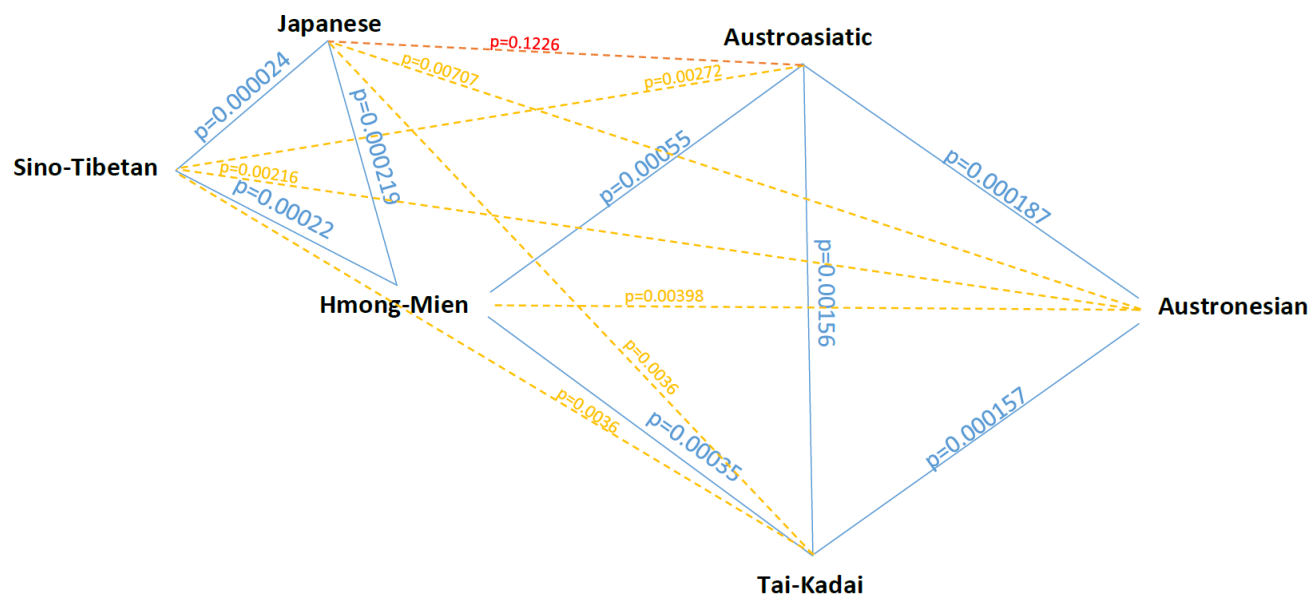

The main elements of the Nostratic and Austric hypothetical macrofamilies appear as the few reliable signals in form of TLF clusters with 4 and more members, which clearly distinguish themselves from the abundant negative signals and statistical noise generated by our analysis of potential links between top-level families of languages (Fig 16). In the final loop of selective analysis, they deliver the best results out of a set of only 11 TLF clusters. Of these 11 clusters, 3 belong to the Nostratic and Austric hypothetical macrofamilies, two contain four core members (Wakashan, Salisham, Iroquaian and Algic), which are part of existing but contested hypotheses: Mosan, 
Almosan and Almosan-Keresiouan. The remaining 6 clusters reflect clearly unrelated TLF connections and as such represent statistical noise in the list, albeit with 5 of them at the bottom of the list when ranked by p-values.

Fig 16. The Nostratic and Austric macrofamilies as inferred by our system

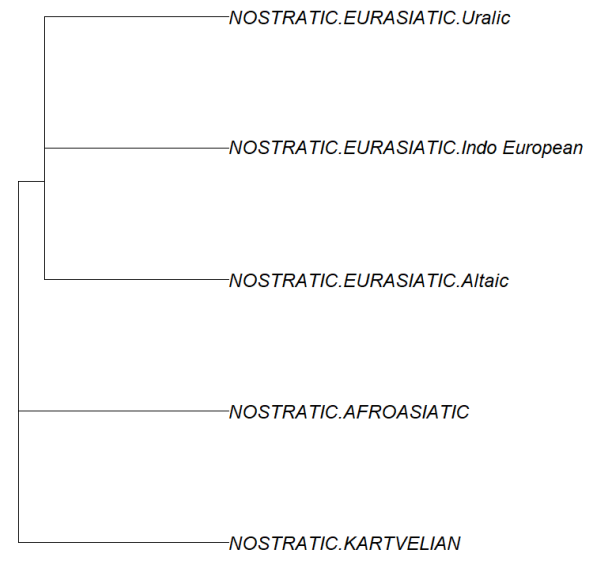

Nostratic

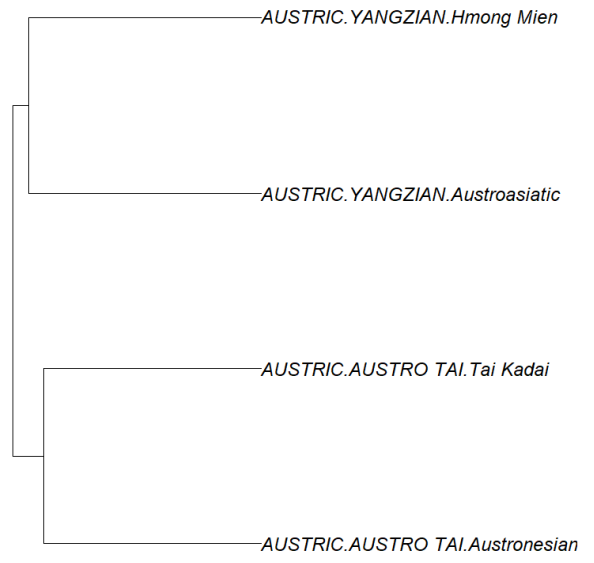

Austric

Fig 17. Inferred macrofamilies and long-range connections: Summary on world map

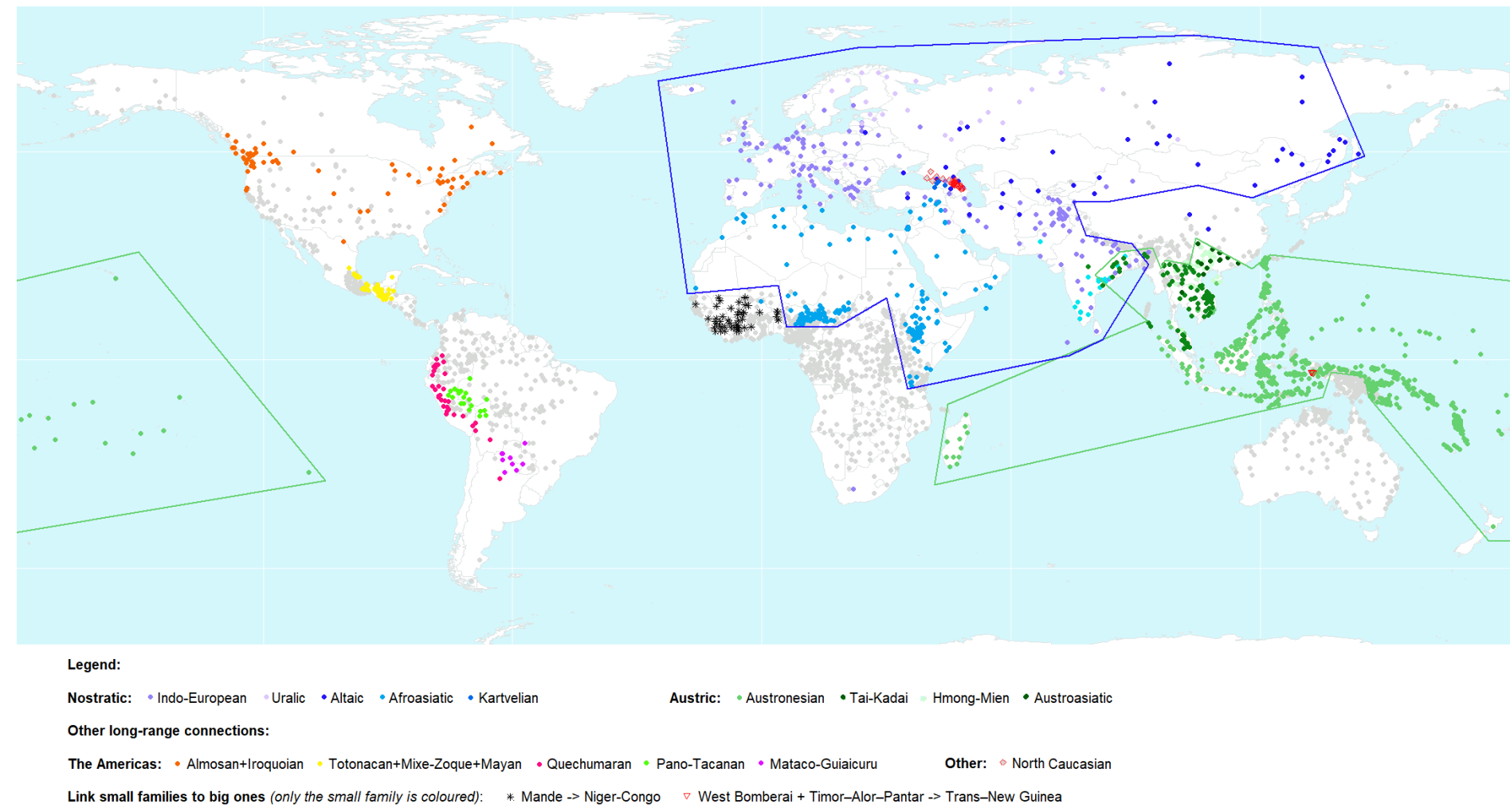


The Americas

The classification of the languages in the Americas leaves the biggest number of primary language families in one continent: 86 in our list, accounting for $23 \%$ of the TLF to TLF pairs. Very few hypotheses that link some of these TLF seem to move in the direction of reaching consensus among scholars. The most comprehensive hypothesis, J.H. Greenberg's Amerind [57] includes all native languages of the Americas in one macrofamily, excluding Na-Dene, Haida and Eskimo-Aleut. This hypothesis has not been welcomed by the community although Greenberg's previous results on the classification of languages in Africa had been successful and is now widely accepted. This situation is reflected in our system, which does not support any wide-scale connection on the American continent but does display a very strong support for the African classifications. Amerind as a whole does not display quantitative signals. However, in the top 40 results with lowest HM p-value in the 3367 TLF to TLF Amerind list, 19 are such for which a long-range hypothesis exists (see Table 7 and Fig 18). It is clear that the last word on the classification of TLFs in the Americas is not spoken and that results in quantitative research shed light on wider connections.

North Caucasian [54]

A connection between the Nakh-Daghestanian and the Northwest Caucasian top-level families is supported by a bootstrap value of 6 in our tree. In the p-value analysis, Northwest Caucasian and Nakh-Daghestanian share an average distance with $\mathrm{p}=0.0024$. This result is still in the range of statistical noise, with 4 unrelated connections to these two families out of 347 exposing a lower p-value.

Other connections

Our TLF to TLF results in the online Supporting Information S7 File can be used to check other hypotheses. Considering only the 16 results with $\mathrm{p}<0.0001$, two other connections unfold which are subject of discussion among scholars: Mande and Niger-Congo (Blench, Dwyer) and the connection between West Bomberai and Trans-New Guinea (Holton and Robinson). This last connection forms with Timor-Alor-Pantar one of the few clusters with all their connections with $\mathrm{p}<0.002$. This is in line with Timothy Usher's current research on New Guinean languages [56].

\section{Discussion}

\section{Materials and methods:}

To make the discussion about our system and its results easier, we provide a graphical overview of the steps involved as well as the different kinds of results the system infers in Fig 18. 
Fig 18. Overview of the processing steps and results of the system

\begin{tabular}{|c|c|c|c|c|c|}
\hline Modeling & Processing & Raw output & $\begin{array}{c}\text { Clustering \& } \\
\text { chance modeling }\end{array}$ & Results validation & Exploration \\
\hline $\begin{array}{l}\text { Word encoding } \\
\text { (Mat.\& meth. I, fig. 1) }\end{array}$ & $\begin{array}{l}\text { Computer processing } \\
\text { (Mat. \& meth. II) }\end{array}$ & $\begin{array}{l}\text { Pairwise distances } \\
\text { (Results I + App. 1) }\end{array}$ & $\begin{array}{l}\text { Phylogeny (S5) } \\
\text { (Mat. \& meth. VII) }\end{array}$ & Challenge against & $\begin{array}{l}\text { Internal classifications: } \\
\text { (III-Subfamiles }+\mathrm{S} 6 \text { ) }\end{array}$ \\
\hline $\begin{array}{l}\text { Best Sound } \\
\text { Change rules (S2) } \\
\text { (Mat. \& meth. III+V) }\end{array}$ & & Distance matrix & $\begin{array}{l}\text { Bootstrapped } \\
\text { matrices } \\
\text { (Mat. \& meth. VI) }\end{array}$ & $\begin{array}{l}\text { (Results II + S4) } \\
\text { Challenge against } \\
\text { chance } \\
\text { (Mat. \& meth. VI-VII) }\end{array}$ & \\
\hline $\begin{array}{l}\text { Point System } \\
\text { (Mat. \& meth. III + V) }\end{array}$ & & $\begin{array}{l}\text { Lists of pairwise } \\
\text { distances }\end{array}$ & $\begin{array}{l}\text { Randomized } \\
\text { languages } \\
\text { (Mat. \& meth. VI) }\end{array}$ & $\begin{array}{l}\text { Bootstrapped } \\
\text { matrices Subfamilies } \\
\text { (III-Subfamilies) }\end{array}$ & $\begin{array}{l}\text { Long-range exploration } \\
\text { (IV-Expl. Long-range+S7) } \\
\text { - Average distances }\end{array}$ \\
\hline $\begin{array}{l}\text { Aligment Rules } \\
(\text { Mat. \& meth. III + V) }\end{array}$ & & & $\begin{array}{l}\text { Pairwise distances } \\
\text { unrelated languages } \\
\text { (Mat. \& meth. VI) }\end{array}$ & $\begin{array}{l}\text { Bootstrapped } \\
\text { matrices Top Level } \\
\text { Families } \\
\text { (IV-Expl. Long-range) }\end{array}$ & $\begin{array}{l}\text { - Harmonic mean p-values } \\
\text { - Bootstrap values }\end{array}$ \\
\hline $\begin{array}{l}\text { Best word list } \\
(\text { Mat. \& meth. } I V+V)\end{array}$ & & & & & \\
\hline & tput & & esult & Su & \\
\hline
\end{tabular}

A first particularity is that modeling the basis elements of the system (sound change rules, word list, etc.) is based primarily on result optimization through error reduction and not on existing findings: tuning the way we modelize the basis elements has an impact on how the system performs with the classification of languages. The principle is the same as for machine learning: training a system with the part of the data where the output is known, avoiding overfitting and inferring a model that performs well with new data.

The second particularity is the sole use of consonants. This choice has also been part of the error reduction process, which showed that not using vowels decreases the perfomance of short-range classifications (internal classifications of young subfamilies like Romance, Slavic or Germanic within Indo-European) but increases the long-range performance. The smaller number of possible vowel clusters compared with the consonants cluster list 59 in use would increase the overall exposure to chance. The exclusive use of consonants also reduces the complexity, allowing us to use simple aligment rules, which were also tuned using the error reduction process. Developing systems that challenge the results against chance is a more accessible task when the models get simpler.

Results:

An important note about the bootstrap values in the phylogenetic trees is that they should always be interpreted as a stability indicator within the very same respective experiments: they are not a confidence value: they just measure how often a result (node) remains stable with the addition of random values in a given context. This stability indicator cannot be used for interpretation across different experiments (e.g. global tree versus subfamily trees). A similar remark has to be repeated regarding p-values, especially Harmonic Mean p-values: they have to be interpreted relatively to each other within the very same analyses.

We find it useful to reflect on three aspects of our results, which offer potential for discussion: 
- The system cannot differentiate the nature of the signals behind the inferred long-range connections: relatedness or early language contact. However, as we can see throughout the research, the word list in use is highly resistant to borrowing.

- Browsing the worldwide phylogeny in the online Supporting Information S5 File we see that well documented languages of Eurasia and Northern Africa (at the bottom of the file) lead to cleaner results. More noise interferes in the classifications of less documented languages like those of New Guinea, Australia or South America. Better quality of the data in use leads to a better differentiation between signals and noise.

\section{Conclusion}

We have presented a new approach for automatic language classification completely based on computer and statistical methods. It reaches a high level of precision, close to human based reference classifications and to one of the best automatic classifications so far available: Gerhard Jäger's one. The system has a strong ability to address the chance issue, not just challenging the results, but also increasing the ability to explore long-range relationships and delivering data for debates about proposed macrofamilies, disputed top-level families and internal classifications.

The top-level families known today arise from the level of visibility we have with the available information and technology in use until now. Opinions diverge on the time scope we can trace back, 8.000 to 10.000 years BC being a widely mentioned limit. Prior to this limit, language history has certainly been as dynamic as thereafter, but most signals of relationships between language families as we use them with classical methods may have disappeared forever. With newer technical possibilities, we can hope to extend the time scope we shed light on. The use of computer techniques for this task is a data science challenge, where our systems deliver signals but also noise. As in most other computer implementations, the system does not replace human expert judgment. The figures alone cannot explain the very complex phenomena behind them. Figures need to be interpreted and wherever possible, linked to other facts: they can support research from other disciplines. Statistical and classical methods are complementary and bring their contribution in interdisciplinary research. Convergence in results deserves a particular attention. In this sense, our results on the Nostratic/Eurasiatic as well as Austric macrofamilies, along with other findings on various distant relationship form a consistent contribution to the debate about deeper relationships between established top-level families of languages.

In the balance between signals and noise, the quality of the data plays a major role. The varying quality of the transcription and word availability, the inhomogeneous record of synonyms, root comparisons versus whole words comparisons as well as the undocumented and unknown loan words are sources of noise, increasing the variance of the results and reducing the precision of the system. Without the tremendous efforts by various institutions and scholars to compile word lists and make them available freely, research like ours would not be possible. The increasing quality of these contributions will be of decisive importance for future improvements of computer-based systems. 


\section{Supporting information}

S1 File. Alignment rules and low points filter Source code of the c\# application which generates the distance matrices. The alignment rules and low signal filters are clearly visible in lines 112 to 231 . osf.io/qs2py/ (C\# as TXT)

S2 File. Sound correspondence Inferred best sound correspondence and point systems with matching probability. osf.io/u2jgx/ (PDF)

S3 File. Conversion ASJP-Our project R script for the conversion of the ASJP source in our format (consonant classes of this project) osf.io/8v9tw/ (R file as TXT)

S4 File. Classification benchmark Benchmark vs. reference classifications (WALS top-level families and Genus, Glottolog, Ethnologue) and Jäger osf.io/pxj3y/(XLS)

S5 File. Worldwide Tree Output phylogeny of the project (raw, bootstrap values) osf.io/3nwqf/ (PNG)

S6 File. Internal classifications Trees of the subfamilies of top-level families (internal classifications, low bootstrap support excluded) osf.io/s86wq/ (PDF)

S7 File. Long-range analysis Pairwise analysis of top-level families osf.io/2rw6a/ (XLS)

S1 Appendix. Pairwise comparisons Query form language to language (WEB)

S2 Appendix. Complete data and scripts osf.io/7g269/files/(WEB)

\section{Acknowledgements:}

We thank Mattis List (Max Planck Institute for the Science of Human History) and Gerhard Jäger (University of Tübingen) for sharing precious inputs and insightful comments.

\section{Author contributions:}

Conceptualization, data curation, software, visualization: Vincent Beaufils Methodology, validation, writing - review \& editing: Vincent Beaufils, Johannes Tomin

\section{References}

1. Starostin, George (ed.) 2011-2019. The Global Lexicostatistical Database. Moscow: Higher School of Economics, \& Santa Fe: Santa Fe Institute. Available online at http://starling.rinet.ru/new100/, accessed on Dec. 27th 2020.

2. Johann-Mattis List, Simon J. Greenhill, and Russell D. Gray "The Potential of automatic word comparison for historical linguistics," PLoS One , 0170046 (2017). 
3. Levinson, S. C., \& Gray, R. D. (2012). Tools from evolutionary biology shed new light on the diversification of languages. Trends in cognitive sciences. 16(3), 167-173. doi:10.1016/j.tics.2012.01.007

4. André Müller, Viveka Velupillai, Søren Wichmann, Cecil H. Brown, Pamela Brown, Eric W. Holman, Dik Bakker, Oleg Belyaev, Dmitri Egorov, Robert Mailhammer, Anthony Grant, and Kofi Yakpo World Language Tree of Lexical Similarity : Version 2 (April 2009) https://asjp.clld.org/static/WorldLanguageTree-002.pdf

5. Jäger, Gerhard Global-scale phylogenetic linguistic inference from lexical resources, Scientific Data, 2018/10/09/online doi:10.1038/sdata.2018.189

6. Trask's Historical Linguistics Revised and edited by Robert McColl Millar, 3rd edition 2015 by Routledge, p 57 doi:10.4324/9781315728056

7. Variation in Indigenous Minority Languages edited by James N. Stanford and Dennis R. Preston doi:10.1111/j.1467-9841.2011.00529_10.x

8. Wichmann, Søren, Eric W. Holman, and Cecil H. Brown (eds.). 2016 The ASJP Database (version 17) https://asjp.clld.org/

9. Ian Maddieson. 2013. Consonant Inventories In: Dryer, Matthew S. \& Haspelmath, Martin (eds.) The World Atlas of Language Structures Online. Leipzig: Max Planck Institute for Evolutionary Anthropology Available online at http://wals.info/chapter/1, Accessed on 2018-02-26.

10. List JM. (2012) SCA: Phonetic Alignment Based on Sound Classes. In: Lassiter D., Slavkovik M. (eds) New Directions in Logic, Language and Computation. ESSLLI 2010, ESSLLI 2011. Lecture Notes in Computer Science, vol 7415. Springer, Berlin, Heidelberg doi:10.1007/978-3-642-31467-4_3

11. Turchin, P., I. Peiros, and M. Gell-Mann (2010) Analyzing genetic connections between languages by matching consonant classes. Journal of Language Relationship 3. 117-126.

12. IPA Chart Available under a Creative Commons Attribution-Sharealike 3.0 Unported License. Copyright 2015 International Phonetic Association. http://www.internationalphoneticassociation.org/content/ipa-chart

13. Gray, R. D., Atkinson, Q. D., \& Greenhill, S. J. (2011) Language evolution and human history: what a difference a date makes. Philosophical Transactions of the Royal Society B: Biological Sciences, 366(1567), 1090-1100. doi: $10.1098 /$ rstb.2010.0378

14. Eska, J. F. (1998). Language history, language change, and language relationship: An introduction to historical and comparative linguistics (p. 116) Hans Henrich Hock, Brian D. Joseph, Language, 74(3), 642-644. doi:10.1353/lan.1998.0094

15. Johann-Mattis List, Mary Walworth, Simon J Greenhill, Tiago Tresoldi, Robert Forkel, Sequence comparison in computational historical linguistics, Journal of Language Evolution, Volume 3, Issue 2, July 2018, Pages 130-144 https://doi.org/10.1093/jole/lzy006

16. List, Johann-Mattis; Greenhill, Simon; Tresoldi, Tiago; and Forkel, Robert (2018) LingPy. A Python library for historical linguistics. Version 2.6.4. http://lingpy.org 
17. List, Johann Mattis \& Rzymski, Christoph \& Greenhill, Simon \& Schweikhard, Nathanael \& Pianykh, Kristina \& Forkel, Robert (eds.) 2019 Concepticon 2.2.0. Jena: Max Planck Institute for the Science of Human History. (Available online at http://concepticon.clld.org)

18. Bynon, T. (1974). Morris Swadesh, The origin and diversification of language, Sherzer Joel (ed.), with a foreword by Dell Hymes. Chicago \& New York: Aldine Atherton, 1971. Pp. xviii + 350. (London: Routledge \& Kegan Paul, 1972.). Journal of Linguistics, 10(1), 153-161. doi:10.1017/s0022226700004072

19. Mark Pagel, Quentin D. Atkinson, Andreea S. Calude, and Andrew Meadea Ultraconserved words point to deep language ancestry across Eurasia PNAS, vol. 110 no. 21, 8471-8476, May 21, 2013. https://www.pnas.org/content/110/21/8471

20. V.M. Illych-Svitych Opyt sravnenya nostraticheskikh jazykov ISBN: 5-354-00173-0 (1971) - "Nauka", 1971-1984.

21. Dolgopolsky Aharon The Nostratic Macrofamily and Linguistic Palaeontology doi:/10.1515/if-2002-0115

22. Josef H. Greenberg Indo-European and its Closest Relatives: The Eurasiatic Language Family Volume II: Lexicon Stanford, CA: Stanford Univ Press; 2002

23. Allan R. Bomhard A comprehensive introduction to Nostratic comparative linguistics, Volume 2 Volume 2, third edition 2018

24. Iaroslav Lebedynsky LES INDO-EUROPÉENS Faits, débats, solutions Editions Errance, ISBN : 978-2-87772-564-4, p. 81

25. Gordon Tullock "A Comment on Daniel Klein's 'A Plea to Economists Who Favor Liberty"' Eastern Economic Journal, Spring 2001

26. Hammarström, Harald \& Forkel, Robert \& Haspelmath, Martin. 2018. Glottolog 3.3. Jena: Max Planck nstitute for the Science of Human History. Available online at http://glottolog.org

27. Daniel J. Wilson The harmonic mean p-value for combining dependent tests Proceedings of the National Academy of Sciences Jan 2019, 116 (4) 1195-1200; DOI: $10.1073 /$ pnas. 1814092116

28. Quentin D. Atkinson, Russell D. Gray Curious Parallels and Curious Connections-Phylogenetic Thinking in Biology and Historical Linguistics, Systematic Biology, Volume 54, Issue 4, August 2005, Pages 513-526 doi:10.1080/10635150590950317

29. Gordon Tullockape package: Paradis E., Claude J. \& Strimmer K. 2004 APE: analyses of phylogenetics and evolution in R language Bioinformatics 20: 289-290

30. R Core Team (2017). R: A language and environment for statistical computing R Foundation for Statistical Computing, Vienna, Austria. https://www.R-project.org/

31. Gascuel, O. (1997) BIONJ: an improved version of the NJ algorithm based on a simple model of sequence data. Molecular Biology and Evolution,14:, 685-695. https://doi.org/10.1093/oxfordjournals.molbev.a025808

32. Josef Finkelstein Confidence Limits Using on Phylogenies: an Approach Using the bootstrap Evolution 39(4), 1985, pp. 783-791 doi:10.1111/j.1558-5646.1985.tb00420.x 
33. Bradley Efron, Elizabeth Halloran, Susan Holmes Bootstrap confidence levels for phylogenetic trees PNAS November 12, 199693 (23) 13429 doi:10.1073/pnas.93.23.13429

34. Dryer, Matthew S. \& Haspelmath, Martin (eds.) 2013 The World Atlas of Language Structures Online. Leipzig: Max Planck Institute for Evolutionary Anthropology. Available online at http://wals.info

35. Eberhard, David M., Gary F. Simons, and Charles D. Fennig (eds.). 2019 Ethnologue: Languages of the World. Twenty-second edition. Dallas, Texas: SIL International. Online version: http://www.ethnologue.com

36. https://glottolog.org/glottolog/glottologinformation

37. Wu, M.-S., N. Schweikhard, T. Bodt, N. Hill, and J.-M. List (2020): Computer-Assisted Language Comparison. State of the Art. Journal of Open Humanities Data 6.2. 1-14. doi:10.5334/johd.12

38. Harmon Luke J, Jason T Weir, Chad D Brock, Richard E Glor, and Wendell Challenger. 2008 GEIGER: investigating evolutionary radiations. Bioinformatics $24: 129-131$

39. Schliep K.P. 2011 Phylogenetic analysis in R Bioinformatics, 27(4) 592-593

40. Jäger, Gerhard. 2018 Extracting language distances and character matrices from ASJP data - Open Science Framework doi:10.17605/OSF.IO/CUFV7

41. Smith MR (2019) Quartet: comparison of phylogenetic trees using quartet and split measures. doi: 10.5281/zenodo.2536318, R package version 1.1.0.

42. Sand A, Holt MK, Johansen J, Brodal GS, Mailund T, Pedersen CNS (2014) tqDist: a library for computing the quartet and triplet distances between binary or general trees. Bioinformatics, 30(14), 2079-2080. doi: 10.1093/bioinformatics/btu157.

43. Smith MR (2019) Bayesian and parsimony approaches reconstruct informative trees from simulated morphological datasets. Biology Letters, 15(2), 20180632. doi: $10.1098 / \mathrm{rsbl} .2018 .0632$.

44. Dediu, Dan. (2018) Making genealogical language classifications available for phylogenetic analysis: Newick trees, unified identifiers, and branch length Language Dynamics and Change. 8. 1-21. doi:10.1163/22105832-00801001

45. Alexandre François Edited by Claire Bowern and Bethwyn Evans

46. BLAZEK, Vaclav. On the Internal Classification of Indo-European Languages: A Survey. Studia Indogermanica Lodziensia, Lodz, 2012, Neuveden, No 7, p. 9-30. ISSN 1506-7254.

47. Blazek, Vaclav. (2013) The Encyclopedia of Global Human Migration DOI: 10.1002/9781444351071.wbeghm815

48. Campbell, Lyle (1997) American Indian languages: the historical linguistics of Native America. Oxford: Oxford University Press. pp. 188, 273-283. ISBN 0-19-509427-1.

49. Kaufman, Terrence (1990) "Language History in South America: What we know and how to know more". In David L. Payne. Amazonian Linguistics. Austin: University of Texas Press. pp. 13-74. 
50. Hammarström, Harald; Forkel, Robert; Haspelmath, Martin, eds. (2017)

"Pano-Tacanan". Glottolog 3.0. Jena, Germany Max Planck Institute for the Science of Human History

51. Gell-Mann, Murray; Ilia Peiros; George Starostin (2009) "Distant Language Relationship: The Current Perspective". Journal of Language Relationship. https://starling.rinet.ru/Texts/distant.pdf

52. Pedro Viegas Barros (2013). La hipótesis de parentesco Guaicurú-Mataguayo: estado actual de la cuestión. Revista Brasileira de Linguística Antropológica, $5.2: 293-333$

53. Cecil H. Brown, David Beck, Grzegorz Kondrak, James K. Watters, and Søren Wichmann "Totozoquean," International Journal of American Linguistics 77, no. 3 (July 2011): 323-372. doi:10.1086/660972

54. Sergei Starostin A NORTH CAUCASIAN ETYMOLOGICALDICTIONARY ASTERISK PUBLISHERS - Moscow 1994

55. Valentin Vydrin (2016) Toward a Proto-Mande reconstruction and an etymological dictionary. Faits de langues, Peter Lang, 2016, Comparatisme et reconstruction : tendances actuelles (Dir. K. Pozdniakov), pp.109-123. halshs-01375776

56. Timothy Usher, Santa Fe Institute https://sites.google.com/site/newguineaworld/

57. Josef H. Greenberg Language in the Americas Stanford, CA: Stanford Univ Press; 1987

58. Starosta, Stanley Proto-East Asian and the origin and dispersal of languages of east and southeast Asia and the Pacific In Sagart, Laurent; Blench, Roger; Sanchez-Mazas, Alicia (eds.). The Peopling of East Asia: Putting Together Archaeology, Linguistics and Genetics. London: Routledge Curzon. pp. 182-197. ISBN 978-0-415-32242-3.

59. Brown, C.H., Holman, E.W., \& Wichmann, S. (2013) Sound Correspondences in the World's Languages. Language 89(1), 4-29. doi:10.1353/lan.2013.0009. 